Commun. math. Phys. 4, 32-63 (1967)

\title{
Derivations and Automorphisms of Operator Algebras
}

\author{
RICHARD V. Kadison* \\ University of Pennsylvania, Philadelphia, Pennsylvania \\ and \\ John R. Ringrose \\ University of Newcastle, Newcastle upon Tyne, England
}

Received July 15, 1966

\begin{abstract}
The theorem that each derivation of a $C^{*}$-algebra $\mathfrak{A}$ extends to an inner derivation of the weak-operator closure $\varphi(\mathfrak{A})^{-}$of $\mathfrak{A}$ in each faithful representation $\varphi$ of $\mathfrak{A}$ is proved in sketch and used to study the automorphism group of $\mathfrak{A}$ in its norm topology. It is proved that the connected component of the identity $\iota$ in this group contains the open ball $\mathscr{B}$ of radius 2 with center $\iota$ and that each automorphism in $\mathscr{B}$ extends to an inner automorphism of $\varphi(\mathfrak{R})^{-}$.
\end{abstract}

\section{Introduction and preliminaries}

Our purpose in this paper is to study the group $\alpha(\mathfrak{U})$ of automorphisms of a $C^{*}$-algebra $\mathfrak{A}$ together with and in relation to some of its subgroups. We note that the mappings $\varphi$ of $C^{*}$-algebras we consider are assumed to preserve adjoints $\left(\varphi\left(A^{*}\right)=\varphi(A)^{*}\right)$ throughout; so that "representation" etc. refer to what is sometimes designated by "*representation" etc. Our particular concern is with $\alpha(\mathfrak{H})$ provided with the topology it acquires from $\mathscr{B}(\mathfrak{A})$, the bounded linear operators on $\mathfrak{A}$ (in its norm), taken in its norm (or, uniform) topology. Recall that each element of $\alpha(\mathfrak{A})$ is an isometry of $\mathfrak{A}[10]$.

In a recent series of papers $[16,18,24]$, it is shown that each derivation of a $C^{*}$-algebra $\mathfrak{A}$ extends to an inner derivation of the weakoperator closure $\mathfrak{A}^{-}$of $\mathfrak{A}$ in every faithful representation of $\mathfrak{A}$. Each such derivation is a bounded linear operator [23] and, as such, the infinitesimal generator of a norm-continuous, one-parameter group of automorphisms of $\mathcal{A}$. The fact that a derivation extends to one which is inner is equivalent to the fact that the automorphisms of the one-parameter group extend to ones which are inner. These considerations as well as an account of the derivation result, for convenience and completness, are found in $\S 2$.

The main technical result of this study (Theorem 7) is that each automorphism of a $C^{*}$-algebra $\mathfrak{A}$ in the interior of the ball $\mathscr{B}$ of radius 2 in $\mathscr{B}(\mathfrak{A})$ with center $\iota$, the identity automorphism of $\mathfrak{A}$, lies on a norm-

\footnotetext{
* Research conducted with the partial support of the NSF and ONR.
} 
continuous one-parameter subgroup of $\alpha(\mathfrak{A})$ and extends to an inner automorphism of $\mathfrak{A}-$ in each faithful representation by virtue of the oneparameter group result. It is proved in the following stages. Each such automorphism $\alpha$ is shown (Lemma 4 ) to extend to an automorphism $\bar{\alpha}$ of $\mathfrak{A}$ - leaving each element of the center of $\mathfrak{A}^{-}$fixed, in each faithful representation of $\mathfrak{A}$, by $C^{*}$-algebra representation and von Neumann algebra methods. (One can go on to show that $\bar{\alpha}$ is spatial at this point, though it is not needed, and follows from the final result.) It is proved (Lemma 5) that each inner automorphism interior to $\mathscr{B}$ of a von Neumann algebra can be implemented by a unitary operator in the algebra with spectrum in an open right half-plane by a combination of von Neumann algebra and spectral theoretic techniques. The next fact (Lemma 6), that each spatial automorphism of a $C^{*}$-algebra which can be implemented by a unitary operator with spectrum in the open right half-plane lies on a norm-continuous one-parameter subgroup of $\alpha(\mathfrak{U})$, is proved by the methods of the theory of analytic operator-valued functions, or $[9$; Corollary 3]. The main theorem (Theorem 7), that the connected component $\gamma(\mathfrak{A})$ of $\iota$ in $\alpha(\mathfrak{A})$ is open, generated (as a group) by one-parameter subgroups of $\alpha(\mathfrak{U})$, and consists of automorphisms which extend to inner automorphism of $\mathfrak{A}^{-}$in each faithful representation of $\mathfrak{A}$, is an easy consequence of these considerations, after passing to the reduced atomic representation. It follows that the various subgroups of $\alpha(\mathfrak{U})$ we consider (with the exception of the group of inner automorphisms) are also open, since they contain the connected component of $\iota$ (by virtue of its "inner" properties). The results of this section (\$3) are in sharp contrast to the situation which obtains if $\alpha(\mathfrak{U})$ is viewed with one of its weaker topologies. As a result of our information in the case of the norm topology, each (norm) continuous representation of a connected topological group in $\alpha(\mathfrak{U})$ has image (in $\gamma(\mathfrak{U})$ ) consisting of automorphisms which extend to inner ones (Corollary 8). On the other hand, Blatwner [1; Corollary] shows that each locally compact group with a countable base has a (faithful) strong-operator continuous representation by unitary operators which induce outer automorphisms of a (hyperfinite) factor of type $I I_{1}$ (except, of course, for the identity operator $I$ ). (N. Suzukr [30] did the same thing for a countable discrete group at the same time.) In [28], SINGER analyzed certain subgroups of $\alpha(\mathfrak{U})$, with $\mathfrak{A}$ a factor of type $I I_{1}$, producing numerous groups of outer automorphisms of $\mathfrak{A}$ in the process. The existence of outer automorphisms of factors of type $I I_{1}$ had been known for some time [6; Exercise 15, p. 308].

In $\S 4$ various special classes of $C^{*}$-algebras and special $C^{*}$-algebras are discussed with regard to their automorphism group and its subgroups to illustrate that all possibilities not in conflict with the results of $\S 3$ can occur for automorphisms on the surface of $\mathscr{B}$ (e.g. they can, in certain 
cases, lie in the connected component of $\iota$; they can, in certain cases, be extendable to be inner in all faithful representations without being either inner or in the connected component of $\iota$, etc.).

In a number of physical contexts, the bounded observables are associated with the self-adjoint operators in a $C^{*}$-algebra $\mathfrak{A}$. The symmetries of the physical system under consideration are expressed in terms of a representation of the physical symmetry group $G$ by automorphisms of $\mathfrak{A}$. In general $G$ will be a Lie group. The infinitesimal generators of the one-parameter subgroups of $G$ often correspond to (unbounded) selfadjoint operators of special physical significance. It is of importance to know whether these generators are observable (in some sense) - equivalently, if the automorphisms corresponding to the one-parameter group are inner. A case in point is the Haag-Araki description of relativistically invariant local quantum fields in terms of von Neumann algebras of bounded local observables. The dynamics and relativistic invariance are expressed in terms of a (strong-operator continuous) unitary representation $g \rightarrow U_{g}$ of the inhomogeneous Lorentz group satisfying certain conditions. The $U_{g}$ induce automorphisms (which are the physically significant entities associated with the $U_{g}$ ) of $\mathfrak{A}$, the $C^{*}$-algebra of (bounded) global observables. The infinitesimal generators of the translation part of $\mathrm{G}$ correspond to the energy and momenta of the field. Given the "spectrum condition" (tantamount to "positive energy"), i.e. that the spectral measure decomposing the representation of the 4-space translation subgroup of $\mathrm{G}$ on its dual group (energy-momentum space) has support in the future light cone of that space; H. Borchers [3] proves that the automorphisms of $\mathfrak{A}$ corresponding to this subgroup extend to inner automorphisms by reducing the unbounded generator case to the bounded one and then applying the norm-continuous representation results. G. DeLL'ANToNio [5], dealing directly with a representation of $G$ by automorphisms satisfying the appropriate analogue of the "positive energy" condition, proves the automorphisms extend to inner ones by making the same reduction to the norm-continuous case. The results of Blattiner, Singer, Suzuki $[1,28,30]$ make it amply clear that something in the nature of the spectrum condition is required to replace norm continuity if "inner" (or "observability") are to be concluded.

We wish to record our gratitude to H. Borchers, G. Dell'Antonio and S. DOPLICHER for their role in discussions of the interplay between the mathematical and physical background of the material in this paper; to J. DIXMIER for pointing out the relevance of [1] to the study of the automorphism group in topologies weaker than the norm topology; and to L. KRISTENSEN for help (specifically noted in $\S 4$ Example d) with certain applications of algebraic topology to groups of automorphisms. Both authors extend their thanks to Professor Svend BundGaARd for the hospitality of the Mathematical Institute in Aarhus during a period of the 
development of these results. The first and second named authors would like to thank Dr. L. Motchane and Professor PaUl Halmos for their kind hospitality at the Institut des Hautes Etudes Scientifiques and at the University of Michigan, respectively, during the initial stages of this work.

We recall that a $C^{*}$-algebra $\mathfrak{A}$ is a Banach algebra with an involution $A \rightarrow A^{*}$ which is a conjugate-linear anti-automorphism of $\mathfrak{A}$ satisfying $\left\|A^{*} A\right\|=\left\|A^{*}\right\| \cdot\|A\|$. Each such $C^{*}$-algebra has a faithful isometric representation as a norm-closed self-adjoint subalgebra of $\mathscr{B}(\mathscr{H})$, the algebra of all bounded operators on a Hilbert space $\mathscr{H}[10,12]$. A state $\varrho$ of $\mathfrak{A}$ is a linear functional on $\mathfrak{A}$ such that $\varrho(I)=1$, where $I$ is the unit element of $\mathfrak{A}$, and $\varrho(A) \geqq 0$ when $A \geqq 0$ (i.e. when the spectrum $\sigma(A)$ of $A$ consists of real non-negative numbers and $\left.A=A^{*}\right)$. Each such $\varrho$ gives rise to a representation $\varphi$ on the completion of the quotient space $2 / \mathscr{K}$ of $\mathfrak{A}$ by the left kernel $\mathscr{K}$ of $\varrho$, the left ideal consisting of those elements $A$ in $\mathscr{A}$ such that $\varrho\left(A^{*} A\right)=0$, relative to the inner product $(A+\mathscr{K}, B+\mathscr{K})$ $=\varrho\left(B^{*} A\right)$, where $\varphi(T)$ is determined by its action on $\mathfrak{Y} / \mathscr{K}$ as $\varphi(T)(A+\mathscr{K})=T A+\mathscr{K}$. From [26] one knows that the pure states, those not expressible as a convex combination of states distinct from it, are precisely the ones which give rise to irreducible representations. In particular, the Krein-Milman theorem [21] yields the fact that there is a separating family of pure states of $\mathfrak{U}$ and, so, a separating family of irreducible representations of $\mathfrak{A}$. Choosing one such representation $\varphi_{s}$ from each equivalence class, we form their direct sum $\varphi$ (where $\varphi(A)$ transforms the vector $\left\{x_{s}\right\}$ in the direct sum of the representation Hilbert spaces onto $\left.\left\{\varphi_{s}(A) x_{s}\right\}\right)$, and refer to this as the reduced atomic representation of $\mathfrak{A}$ ("the" since any other such is unitarily equivalent to it).

Definition. An automorphism $\alpha$ of a $C^{*}$-algebra $\mathfrak{A}$ acting on a Hilbert space $\mathscr{H}$ is said to be: extendable if there is an automorphism of the weakoperator closure of $\mathfrak{A}$ equal to it on $\mathfrak{A}$, spatial if there is a unitary operator $U$ on $\mathscr{H}$ such that $\alpha(A)=U A U^{*}$ for each $A$ in $\mathfrak{A}$, and weakly-inner if it is spatial and $U$ can be chosen in the weak-operator closure of $\mathfrak{A}$. If $\varphi$ is a faithful representation of $\mathfrak{A}$ on a Hilbert space, we denote by $\varepsilon_{\varphi}(\mathfrak{A})$, $\sigma_{\varphi}(\mathfrak{R})$, and $\iota_{\varphi}(\mathfrak{Q})$, the groups of those elements $\alpha$ of the automorphism group of $\mathfrak{A}$ for which $\varphi \propto \varphi^{-1}$ is extendable, spatial, and weakly-inner, respectively. We denote by $\pi(\mathfrak{R})$ the intersection of all the subgroups $\iota_{\varphi}(\mathfrak{H})$ and refer to its elements as permanently weakly (for brevity, $\pi$-) inner automorphisms of $\mathfrak{A}$. We write $\iota_{0}(\mathfrak{H})$ for the group of inner automorphisms of $\mathfrak{A}$ and $\gamma(\mathfrak{A})$ for the connected component of $\iota$ in $\alpha(\mathfrak{A})$ provided with its norm topology.

The $\pi$-inner automorphisms of $\mathfrak{A}$ would seem to be the "eternal" symmetries of the physical system $\mathfrak{A}$ represents. We note, especially, that there are such symmetries (in $\gamma(\mathfrak{Z})$ ) which are not inner and such symmetries which are neither inner nor in $\gamma(\mathfrak{A})$. 


\section{Derivations and inner automorphisms}

We present a brief survey of the proof that each derivation of a von Neumann algebra is inner. To begin with, note that each derivation $\delta$ of a $C^{*}$-algebra $\mathfrak{A}$ acting on the Hilbert space $\mathscr{H}$ is continuous on the unit ball $\mathscr{S}_{1}$ of $\mathscr{A}$ taken in the weak operator topology. For this one makes use of SAKAI's result that $\delta$ is norm continuous [23], the equality $(\delta(A) x, y)=\left(\delta\left(A^{\frac{1}{2}}\right) A^{\frac{1}{2}} x, y\right)+\left(A^{\frac{1}{2}} \delta\left(A^{\frac{1}{2}}\right) x, y\right)$ for $A \geqq 0$ and the strongoperator continuity of $A \rightarrow A^{\frac{1}{2}}$ on the set of positive bounded operators. This establishes the continuity of $\delta$ on the positive elements in $\mathscr{S}_{\mathbf{1}}$ at 0 from $\mathscr{S}_{1}$ taken in the strong-operator topology to $\mathfrak{A}$ taken in the weakoperator topology. The strong-operator continuity of $A \rightarrow A^{+}$and $A \rightarrow A^{-}$on the self-adjoint operators together with $A=A^{+}-A^{-}$and this last conclusion yields the same continuity of $\delta$ at 0 on the selfadjoint operators in $\mathscr{S}_{1}$. The linearity of $\delta$ yields this continuity on the self-adjoint operators in $\mathscr{S}_{1}$, and this linearity together with the fact that the weak and strong-operator closures of a convex set of operators coincide give the continuity of $\delta$ on the self-adjoint operators in $\mathscr{S}_{1}$ taken in the weak-operator topology. The weak-operator continuity of the adjoint mapping and the decomposition $A=\left(A+A^{*}\right) / 2+$ $+i\left(A-A^{*}\right) / 2 i$ give the same continuity for $\delta$ on $\mathscr{S}_{\mathbf{1}}$.

It follows, next, that $\delta$ extends to the weak-operator closure $\mathscr{S}_{1}^{-}$of $\mathscr{S}_{1}$ and then linearly to $\mathfrak{A}^{-}$the weak-operator closure of $\mathfrak{A}$, a von Neumann algebra. The extension $\bar{\delta}$ so obtained is a derivation of $\mathfrak{A}^{-}$. Let $\mathscr{A}$ be a (self-adjoint) maximal abelian subalgebra of $\mathfrak{A}^{\prime}$, the commutant of $\mathfrak{A}$ (the existence of such an $\mathscr{A}$ is easily established by the use of Zorn's lemma); and let $\mathscr{P}$ be the lattice of orthogonal projection operators in $\mathscr{A}$. With $\mathfrak{A}_{0}$ the set $\left\{A_{1} E_{1}+\cdots+A_{n} E_{n}: A_{1}, \ldots, A_{n}\right.$ in $\mathfrak{H}^{-}$and $E_{1}, \ldots, E_{n}$ in $\mathscr{P}\}$, define $\delta_{0}$ on $\mathfrak{A}_{0}$ by:

$$
\delta_{0}\left(A_{1} E_{1}+\cdots+A_{n} E_{n}\right)=\bar{\delta}\left(A_{1}\right) E_{1}+\cdots+\bar{\delta}\left(A_{n}\right) E_{n} .
$$

One establishes that $\delta_{0}$ is well-defined (i.e. independent of the representation of an operator in the form $A_{1} E_{1}+\cdots+A_{n} E_{n}$ ), is a derivation of the self-adjoint operator algebra $\mathfrak{A}_{0}$ into $\mathfrak{A}_{0}$ and is bounded. From the boundedness and linearity of $\delta_{0}$ it extends to a derivation of the norm closure of $\mathfrak{A}_{0}$, a $C^{*}$-algebra. From the preceding, this extension has, in turn, an extension $\bar{\delta}_{0}$ to the von Neumann algebra $\mathfrak{A}_{0}^{-}$. Since $\mathfrak{A}_{0}^{-}$contains $\mathfrak{A}$, its commutant $\mathfrak{A}_{0}^{\prime}$ is contained in $\mathfrak{A}^{\prime}$; and since $\mathfrak{A}_{0}$ contains $\mathscr{P}, \mathfrak{A}_{0}^{-}$ contains $\mathscr{A}$ and $\mathfrak{A}_{0}^{\prime}$ commutes with $\mathscr{A}$. But $\mathscr{A}$ is maximal abelian in $\mathfrak{A}^{\prime}$; so that $\mathfrak{A}_{0}^{\prime}$ is contained in $\mathscr{A}$ and is abelian. Thus $\mathfrak{A}_{0}^{-}$is a von Neumann algebra of type $I$, and from [20; Theorem 9], $\bar{\delta}_{0}$ is inner. Say $\bar{\delta}_{0}(A)$ $=B A-A B$, with $B$ in $\mathfrak{A}_{0}^{-}$, for all $A$ in $\mathfrak{A}_{0}^{-}$. Since $\bar{\delta}_{0}(E)=0(=\delta(I) E)$ for each $E$ in $\mathscr{P}, B$ commutes with $\mathscr{A}$. Moreover, since $\delta_{0}$ is an extension 
of $\delta$, we have that $\delta$ is spatial (i.e. of the form $A \rightarrow B A-A B=\operatorname{ad} B(A)$ for some bounded operator $B$ ).

The remainder of the argument consists of showing that $B$ can be chosen in $\mathfrak{A}-$. If $\delta(A)=B_{0} A-A B_{0}$ for each $A$ in $\mathfrak{A}$ then $B-B_{0}$ lies in $\mathfrak{A}^{\prime}$. Conversely $\left(B+B^{\prime}\right) A-A\left(B+B^{\prime}\right)=\delta(A)$ for each $A$ in $\mathfrak{A}$ and $B^{\prime}$ in $\mathfrak{A}^{\prime}$. If $U^{\prime}$ is a unitary operator in $\mathfrak{I}^{\prime}, U^{\prime *} B U^{\prime} A-A U^{\prime *} B U^{\prime}$ $=B A-A B$, so that each operator in $\operatorname{co}_{\mathcal{A}^{\prime}}(B)$, the convex hull of $\left\{U^{\prime *} B U^{\prime}: U^{\prime}\right.$ a unitary operator in $\left.\mathfrak{l}^{\prime}\right\}$, and in $\overline{\operatorname{co}}_{\mathfrak{H}^{\prime}}(B)$, its weakoperator closure gives rise to $\delta$ on $\mathfrak{A}$. Now $\overline{\mathrm{co}}_{\mathfrak{A}},(B)$ is weak-operator compact, convex, non-null and stable under the mappings $T \rightarrow U^{\prime *} T U^{\prime}, U^{\prime}$ a unitary operator in $\mathfrak{A}^{\prime}$. Zorn's lemma provides a minimal such subset $\mathscr{K}$ of $\overline{\mathrm{co}}_{\mathfrak{A}^{\prime}}(B)$. One establishes, now, that $\mathscr{K}$ consists of a single element which, by stability under the mappings $T \rightarrow U^{\prime *} T U^{\prime}$, commutes with all the unitary operators in $\mathfrak{A}^{\prime}$, hence with all operators in $\mathfrak{A}^{\prime}$; and, therefore, lies in $\mathfrak{A}^{-}$. Since $\mathscr{K}$ is minimal, $\left\|B_{1} P\right\|=\left\|B_{2} P\right\|$ for each $B_{1}$ and $B_{2}$ in $\mathscr{K}$ and each operator $P$ in the center $\mathscr{C}$ of $\mathfrak{A}-\left(\right.$ for $\left\{B_{0}: B_{0} \in \mathscr{K}\right.$ and $\left.\left\|B_{0} P\right\| \leqq a\right\}$ is convex, weak-operator compact and stable under the mappings $T \rightarrow U^{\prime *} T U^{\prime}$ ). Since $B$ commutes with $\mathscr{A}$ and $\mathscr{A}$ contains $\mathscr{C}$, $B$ and hence each $B_{0}$ giving rise to $\delta$ commutes with $\mathscr{C}$. Thus the argument may be given assuming $\mathfrak{A}-$ to be of pure type. We illustrate the rest of the argument in the case where $\mathfrak{A}$ - is of type $I I I$. (The other cases involve some variations of this argument, though one could deal just with the type $I I I$ case by using a device of SAKAI [24]. The algebra $\mathfrak{A}$ - is tensored with a factor of type $I I I$ and $\delta$ is extended to this product, an algebra of type $I I I$ by [22], as we did in defining $\delta_{0}$. It is easy to show that the extension is inner if and only if $\delta$ is.)

Assuming $\mathfrak{A}-$ is of type $I I I$ let $\mathscr{K}_{0}$ be the set of differences of operators in $\mathscr{K}$. Then $\mathscr{K}_{0}$ is a subset of $\mathfrak{U}^{\prime}$, is weak-operator compact, convex, non-null and stable under the mappings $T \rightarrow U^{\prime *} T U^{\prime}$. Of course, we want to show that $\mathscr{K}_{0}$ consists of 0 alone. Since $B^{*} A-A B^{*}$ $=-\left(B A^{*}-A^{*} B\right)^{*}$ is in $\mathfrak{A}$, for each $A$ in $\mathfrak{A}, B+B^{*}$ and $B-B^{*}$ provide derivations of $\mathfrak{A}$; so that we may assume, at the outset that $B$ is self-adjoint. Replacing $B$ by $B+\|B\| I$, we may assume, moreover, that $B \geqq 0$. Then each element of $\overline{\mathrm{co}}_{\mathcal{H}^{\prime}}(B)$ is positive. If $A_{0}$ in $\mathscr{K}_{0}$ is not 0 , the lemma following this discussion, which is a slight extension of J. Schwartz's slight extension [25; XXII p. 3.33, Lemma 15] of the Dixmier Process [6: Chapter $3, \S 5]$, implies that $\overline{\mathrm{co}}_{\mathcal{H}^{\prime}}\left(A_{0}\right)$ contains a non-zero central operator $C$. Since $-A_{0}$ lies in $\mathscr{K}_{0}$ so does $-C$. For at least one of $C$ and $-C$, say $C$, there is an $a>0$ and a central projection $P$ such that $C P>a P$. Now $C=B_{1}-B_{2}$, for some $B_{1}$ and $B_{2}$ in $\mathscr{K}$; and $\left\|B_{1} P\right\|$ $=\left\|B_{2} P\right\|=\left\|B_{2} P+C P\right\| \geqq\left\|B_{2} P+a P\right\|>\left\|B_{2} P\right\|$ (since $B_{2} P \geqq 0$ ), a contradiction. Thus $\mathscr{K}_{0}$ contains only $0, \mathscr{K}$ has a single element in $\mathfrak{A}^{-}$ inducing $\delta$, and $\delta$ on $\mathfrak{A}^{-}$is inner. 
We may assume, in the foregoing that $\mathfrak{A}^{\prime}$ is countably decomposable, for if $\left\{P_{\alpha}\right\}$ is an orthogonal family of central projections, $\delta\left(P_{\alpha}\right)=0$ as noted; so that $\delta$ maps $\mathfrak{A}-P_{\alpha}$ into itself. If this derivation is inner and induced by $B_{\alpha}$ with $\left\|B_{\alpha}\right\| \leqq\|B\|$, then $\Sigma B_{\alpha}$ induces $\delta$ on $\mathfrak{H}^{-}$and lies in $\mathfrak{A}^{-}$. Using projections in $\mathscr{C}$ cyclic under $\mathscr{C}^{\prime}$, we may assume $\mathscr{C}$ is countably decomposable. In this case $\mathfrak{A}^{\prime}$ has a cyclic projection $E^{\prime}$ with central carrier $I$. Since $A \rightarrow A E^{\prime}$ is an isomorphism of $\mathfrak{A}^{-}$with $\mathfrak{A}^{-} E^{\prime}$, we may work with $\mathfrak{A}-E^{\prime}$, whose commutant $E^{\prime} \mathfrak{U}^{\prime} E^{\prime}$ is countably decomposable. With this in mind, the lemma following is the extension of the Dixmier Process needed in our argument.

Lemma 1. If $\mathscr{R}$ is a countably decomposable von Neumann algebra of type III, then $\operatorname{co}_{\mathscr{R}}(A)$ has a non-zero operator from the center $\mathscr{C}$ of $\mathscr{R}$ in its norm closure if $A$ is a non-zero element of $\mathscr{R}$.

Proof. With $\mathscr{F}$ a family of operators, we say that the positive linear mapping $\alpha$ defined by $\alpha(B)=\sum_{j=1}^{n} a_{j} U_{j}^{*} B U_{j}$ with $a_{j} \geqq 0, \Sigma a_{j}=1$ and each $U_{j}$ a unitary operator is from $\mathscr{F}$ when each $U_{j}$ lies in $\mathscr{F}$. Note that $\|\alpha\| \leqq 1$ and that, if $\alpha$ is from an algebra of operators with center $\mathscr{C}$, $\alpha(C)=C$ for each $C$ in $\mathscr{C}$.

If we can prove:

for each non-zero $A$ in $\mathscr{R}$ and each $\varepsilon$ in $(0,1)$ there is an $\alpha$ from

$\mathscr{R}$ and $C$ in $\mathscr{C}$ such that $\|\alpha(A)-C\|<\varepsilon\|C\|-$ if $A$ is self-adjoint,

$C$ may be chosen self-adjoint and such that $\|A\| \leqq(1+\varepsilon)\|C\|$; then, given non-zero $A$ in $\mathscr{R}$, we may choose $\alpha_{1}, \alpha_{2}, \ldots$ from $\mathscr{R}$ and $C_{1}, C_{2} \ldots$ in $\mathscr{C}$ such that

Hence

$$
\left\|\alpha_{n} \alpha_{n-1} \ldots \alpha_{1}(A)-C_{n}\right\|<(n+1)^{-1}\left\|C_{n}\right\| .
$$

and with $m>n$,

$$
n(n+1)^{-1}\left\|C_{n}\right\| \leqq\left\|\alpha_{n} \ldots \alpha_{1}(A)\right\| \leqq\|A\| ;
$$

$$
\begin{aligned}
\left\|C_{m}-C_{n}\right\| & \leqq\left\|C_{m}-\alpha_{m} \ldots \alpha_{1}(A)\right\|+\left\|\alpha_{m} \ldots \alpha_{1}(A)-C_{n}\right\| \leqq \\
& \leqq(m+1)^{-1}\left\|C_{m}\right\|+\left\|\alpha_{n} \ldots \alpha_{1}(A)-C_{n}\right\| \leqq m^{-1}\|A\|+n^{-1}\|A\|< \\
& <2 n^{-1}\|A\| .
\end{aligned}
$$

Thus $\left\{C_{n}\right\}$, and therefore also $\left\{\alpha_{n} \ldots \alpha_{1}(A)\right\}$, converge to some $C_{0}$ in $\mathscr{C}$. Since

$\left\|C_{0}-C_{1}\right\|=\lim _{n \rightarrow \infty}\left\|\alpha_{n} \ldots \alpha_{1}(A)-C_{1}\right\| \leqq\left\|\alpha_{1}(A)-C_{1}\right\|<\frac{1}{2}\left\|C_{1}\right\|$,

$C_{0}$ is non-zero and the lemma follows.

It remains to prove $(*)$. Given $(*)$ for self-adjoint operators, if $A=A_{1}+i A_{2}$ with, say, $A_{1}$ non-zero and $A_{1}, A_{2}$ self-adjoint, choose $C_{1}$ and $C_{2}$ self-adjoint in $\mathscr{C}$ and $\alpha_{1}, \alpha_{2}$ from $\mathscr{R}$ such that

$\left\|\alpha_{1}\left(A_{1}\right)-C_{1}\right\|<\frac{1}{2} \varepsilon\left\|C_{1}\right\|, \quad\left\|\alpha_{2} \alpha_{1}\left(A_{2}\right)-C_{2}\right\| \leqq \frac{1}{2} \varepsilon\left\|C_{2}\right\|$. 
Then $\left\|\alpha_{2} \alpha_{1}(A)-C\right\|<\varepsilon\|C\|$, where $C=C_{1}+i C_{2}$. We may confine attention to a non-zero, self-adjoint $A$ in $\mathscr{R}$. Given $\varepsilon>0$, we can find orthogonal (spectral) projections $E_{1}, \ldots, E_{n}$ and real numbers $a_{1}, \ldots, a_{n}$ such that $\left\|A-\Sigma a_{j} E_{j}\right\|<\frac{1}{3} \varepsilon\|A\|$ and $\max \left|a_{j}\right|=\|A\|$. If $(*)$ holds for such a sum of projections, choose $C$ self-adjoint in $\mathscr{C}$ and $\alpha$ from $\mathscr{R}$ such that

$$
(1+\varepsilon)\|C\| \geqq\left\|\Sigma a_{j} E_{j}\right\|=\|A\| \text { and }\left\|\alpha\left(\sum a_{j} E_{j}\right)-C\right\|<\frac{1}{3} \varepsilon\|C\| \text {. }
$$

Then

$$
\begin{aligned}
&\|\alpha(A)-C\| \leqq\left\|\alpha(A)-\alpha\left(\sum a_{j} E_{j}\right)\right\|+\left\|\alpha\left(\sum a_{j} E_{j}\right)-C\right\| \\
& \leqq \frac{1}{3} \varepsilon(\|A\|+\|C\|) \leqq \frac{(2+\varepsilon) \varepsilon}{3}\|C\| \leqq \varepsilon\|C\| .
\end{aligned}
$$

Note next that there are mutually orthogonal projections $Q_{1}, \ldots, Q_{m}$ in $\mathscr{C}$ such that $Q_{k} E_{j}$ and $Q_{k}-\sum_{j} Q_{k} E_{j}$ have central carrier $Q_{k}$ or 0 for each $j$ and $k, Q_{k} \Sigma_{j} a_{j} E_{j} \neq 0$ for each $k$, and $\left(\Sigma_{k} Q_{k}\right)\left(\sum_{j} a_{j} E_{j}\right)=\Sigma_{j} a_{i} E_{j}$. If (*) holds for $\Sigma_{j} a_{j} E_{j} Q_{k}$ for each $k$, choose $\beta_{k}$ from $\mathscr{R} Q_{k}$ and $C_{k} Q_{k}$ selfadjoint in $\mathscr{C} Q_{k}$, the center of $\mathscr{R} Q_{k}$ such that $\left\|\beta_{k}\left(\Sigma_{j} a_{j} E_{j} Q_{k}\right)-C_{k} Q_{k}\right\|<$ $<\varepsilon\left\|C_{k} Q_{k}\right\|$ and $\left\|\Sigma_{j} a_{j} E_{j} Q_{k}\right\| \leqq(1+\varepsilon)\left\|C_{k} Q_{k}\right\|$. Defining $\alpha_{k}$ on $\mathscr{R}$ as the linear extension of $\beta_{k}$ on $\mathscr{R} Q_{k}$ and the identity on $\mathscr{R}\left(I-Q_{k}\right), \alpha_{k}$ is from $\mathscr{R}$, satisfies the same inequality as $\beta_{k}$ and

In addition,

$$
\left\|\alpha_{1} \ldots \alpha_{m}\left(\Sigma_{j} a_{j} E_{j}\right)-\Sigma_{k} C_{k} Q_{k}\right\|<\varepsilon\left\|\Sigma_{k} C_{k} Q_{k}\right\| .
$$

$$
\left\|\Sigma_{j} a_{j} E_{j}\right\| \leqq(1+\varepsilon)\left\|\Sigma_{k} C_{k} Q_{k}\right\| .
$$

These reductions permit us to assume that $A=\sum_{j=1}^{n} a_{j} E_{j}$, that each $E_{j}$ has central carrier $Q$, that $Q-\Sigma E_{j}\left(=F_{m}\right)$ is either 0 or has central carrier $Q$, and that $\|A\|=\left|a_{n}\right|$. Since $\mathscr{R}$ is of type $I I I$ and countably decomposable, all the $E_{j}$ are equivalent. Moreover, $E_{n}$ is the sum of projections $F_{n}, F_{n+1}, \ldots, F_{m-1}$, for $m$ arbitrarily large, each equivalent to $E_{1}$. Writing $F_{j}$ for $E_{j}$ with $j<n, b_{j}$ for $a_{j}$ with $j \leqq n, b_{j}$ for $a_{n}$ with $n<j<m, b_{m}$ for 0 and $m^{\prime}$ for $m-1$ or $m$ according as $F_{m}$ is or is not 0 , we have $\sum_{j=1}^{n} a_{j} E_{j}=\sum_{j=1}^{m^{\prime}} b_{j} F_{j}$. Choosing suitable partial isometries in $\mathscr{R}$ between the $F_{j}$ 's, we can construct a unitary operator $U_{\tau}$ in $\mathscr{R}$ such that $U_{\tau}^{*}\left(\sum_{j=1}^{m^{\prime}} b_{j} F_{j}\right) U_{\tau}=\sum_{j=1}^{m^{\prime}} b_{j} F_{\tau(j)}$, for each permutation $\tau$ of $\left\{1, \ldots, m^{\prime}\right\}$. With $S$ the group of all permutations of $\left\{1, \ldots, m^{\prime}\right\}$ and $\alpha_{m}$ from $\mathscr{R}$ defined by $\alpha_{m}(B)=\frac{1}{m^{\prime} !} \sum_{\tau \text { in } S} U_{\tau}^{*} B U_{\tau}$, we have $\alpha_{m}\left(\sum_{j=1}^{m^{\prime}} b_{j} F_{j}\right)=b Q$, where $b=\frac{1}{m^{\prime}} \sum_{j=1}^{m^{\prime}} b_{j}$ $=\frac{1}{m^{\prime}} \sum_{j=1}^{n-1} a_{j}+\frac{m-n}{m^{\prime}} a_{n}$. With $C=a_{n} Q$, we have $\|A\|=\|C\|$. Since $n$ is fixed, given $\varepsilon>0$, we can choose $m$ so large that $\left\|\alpha_{m}\left(\Sigma a_{j} E_{j}\right)-C\right\|<\varepsilon\|C\|$. 
The result on derivations of $C^{*}$-algebras can be rephrased in terms of one-parameter groups of automorphisms. In this form it is the key lemma of our study, though its conclusion is subsumed in Corollary 8.

Lemma 2. If $t \rightarrow \alpha(t)$ is a norm-continuous one-parameter group of (i.e. representation of the additive group of reals by) automorphisms of a $C^{*}$-algebra $\mathfrak{A}$ acting on a Hilbert space $\mathscr{H}$ then each $\alpha(t)$ is weakly-inner.

Proof. From [8; Theorem 2, p. 614], there is a bounded linear operator $\delta$ on $\mathfrak{A}$ such that $\exp t \delta=\alpha(t)$ for each real $t$ ( $\delta$ is the infinitesimal generator of $t \rightarrow \alpha(t))$. The series for $\exp t \delta$ yields

$$
\begin{aligned}
\alpha(t)[A B] & =A B+t \delta(A B)+O\left(t^{2}\right)=\alpha(t)[A] \alpha(t)[B] \\
& =A B+t(A \delta(B)+\delta(A) B)+O\left(t^{2}\right),
\end{aligned}
$$

so that $\delta$ is a derivation. The derivation theorem tells us that $\delta=\operatorname{ad} i A \mid \mathfrak{A}$, with $A$ in $\mathfrak{A}^{-}$(and $A=A^{*}$, since $\delta\left(B^{*}\right)=\delta(B)^{*}$ for each $B$ in $\mathfrak{A}$ ). Comparing series coefficients $\alpha(t)[B]=(\exp t \delta)(B)=U_{t} B U_{-t}$, with $U_{t}(=\exp i t A)$ a unitary operator in $\mathfrak{A}^{-}$.

\section{The automorphism group}

The principal results are contained in this section.

Lemma 3. If $\alpha$ is an automorphism of a $C^{*}$-algebra $\mathfrak{A}$ acting on a Hilbert space and $\alpha$ is weak-operator bicontinuous on the unit ball of $\mathfrak{A}$ (i.e. $\alpha$ is ultra-weakly bicontinuous on $\mathfrak{A})$ then $\alpha$ has an extension $\bar{\alpha}$ which is an automorphism of $\mathfrak{A}^{-}, \bar{\alpha}$ is ultra-weakly bicontinuous on $\mathfrak{A}^{-}$, and $\|\bar{\alpha}-\iota\|=\|\alpha-\iota\|$.

Proof. From [17; Lemma (2.3)], $\alpha$ has an ultra-weakly continuous extension $\bar{\alpha}$ to $\mathfrak{A}^{-}$with image $\mathfrak{A}^{-}$. The argument of [17; Lemma (2.4)] shows that $\bar{\alpha}$ is a homomorphism. The same considerations applied to $\alpha^{-1}$ yield an ultra-weakly continuous mapping of $\mathfrak{A}^{-}$onto $\mathfrak{A}^{-}$inverse to $\bar{\alpha}$ on $\mathfrak{A}$. By ultra-weak continuity, this mapping is inverse to $\bar{\alpha}$ on $\mathfrak{A}^{-}$; so that $\bar{\alpha}$ is an automorphism of $\mathfrak{A}^{-}$. From the Kaplansky density theorem, the unit ball of $\mathfrak{A}$ is strong (hence, weak)-operator dense in that of $\mathfrak{A}^{-}$; so that the ultra-weakly continuous mapping $\bar{\alpha}-\imath$ maps the unit ball of $\mathfrak{A}^{-}$into the weak-operator closure of the image under $\bar{\alpha}-\imath$ of the unit ball of $\mathfrak{A}$. This closure is contained in the closed ball of radius $\|\alpha-\iota\|$ in $\mathfrak{A}^{-}$. Thus $\|\bar{\alpha}-\iota\| \leqq\|\alpha-\iota\|$; and, of course, $\|\bar{\alpha}-\iota\|$ $=\|\alpha-\imath\|$.

Lemma 4. If $\alpha$ is an automorphism of a $C^{*}$-algebra $\mathfrak{A}$ acting on a Hilbert space, and $\|\alpha-\imath\|<2$, then $\alpha$ extends to an automorphism $\bar{\alpha}$ of $\mathfrak{A}^{-}$, leaving each element of the center of $\mathfrak{A}^{-}$fixed, such that $\|\bar{\alpha}-\imath\|=\|\alpha-\imath\|$.

Proof. Suppose that $\mathfrak{A}$ acts on the Hilbert space $\mathscr{H}$, that $\alpha$ is an automorphism of $\mathfrak{A}$ and that $\|\alpha-\imath\|<2$. With $E^{\prime}$ a projection in $\mathfrak{A}^{\prime}$, and $\varphi$ defined by $\varphi(A)=\alpha(A) E^{\prime}$, for $A$ in $\mathfrak{A},(\varphi \oplus \iota)(\mathfrak{A})$ acting on $\mathscr{H} \oplus E^{\prime}(\mathscr{H})$ 
does not have strong-operator closure $\varphi(\mathfrak{Q})-\oplus \mathfrak{A}-$. Otherwise there is an $A$ in the unit ball of $\mathfrak{A}$, with $x$ a unit vector in $E^{\prime}$ given, such that

$$
\begin{aligned}
1-\frac{1}{2}\|\alpha-\imath\|>\|\left[(\varphi \oplus \iota)(A)-\left(-E^{\prime} \oplus I\right)\right] & (x, x) \| \\
& =\left\|\left(\alpha(A) E^{\prime} x, A x\right)-(-x, x)\right\|
\end{aligned}
$$

and

$$
\|A x-x\|<1-\frac{1}{2}\|\alpha-\imath\|, \quad\|\alpha(A) x+x\|<1-\frac{1}{2}\|\alpha-\imath\| .
$$

Hence

$$
\|\alpha-\imath\| \geqq\|\alpha(A) x-A x\|>\|2 x\|-2\left(1-\frac{1}{2}\|\alpha-\imath\|\right)=\|\alpha-\imath\| .
$$

It follows now from [12; Lemma 3] that $\varphi$ and $\iota$ are not disjoint representations of $\mathfrak{A}$. Zorn's lemma provides us with a maximal orthogonal family $\left\{F_{a}^{\prime}\right\}$ of projections in $\mathfrak{A}^{\prime}$ such that, for each $F_{a}^{\prime}$ there is a projection $G_{a}^{\prime}$ in $\mathfrak{A}^{\prime}$ and a partial isometry $U_{a}$ with initial space $G_{a}^{\prime}$ and final space $F_{a}^{\prime}$ such that $\alpha(A) F_{a}^{\prime}=U_{a} A G_{a}^{\prime} U_{a}^{*}$. Maximality of $\left\{F_{a}^{\prime}\right\}$ and the fact that $\varphi$ and $\iota$ are not disjoint no matter which (non-zero) projection $E^{\prime}$ we use in defining $\varphi$, allows us to conclude that $\Sigma_{a} F_{a}^{\prime}=I$. Thus $\alpha(A)=\Sigma_{a} U_{a} A G_{a}^{\prime} U_{a}^{*}$ for all $A$ in $\mathfrak{A}$. With $y$ and $z$ vectors in $\mathscr{H}$, there is a finite subset $F_{1}^{\prime}, \ldots, F_{m}^{\prime}$ of $\left\{F_{a}^{\prime}\right\}$ such that $\left\|y-\sum_{j=1}^{m} F_{j}^{\prime} y\right\|<1 / 4\|z\|$. If $A$ and $B$ in the unit ball of $\mathfrak{A}$ are such that $\left|\left([A-B] G_{j}^{\prime} U_{j}^{*} y, U_{j}^{*} z\right)\right|<1 / 2 m$ for $j=1, \ldots, m$; remembering that $\alpha$ is isometric on $\mathfrak{A}$,

$$
\begin{aligned}
|(\alpha(A-B) y, z)| \leqq \mid(\alpha(A-B) & \left.\left(\Sigma F_{j}^{\prime} y\right), z\right) \mid+2\left\|y-\Sigma F_{j}^{\prime} y\right\| \cdot\|z\| \leqq \\
& \leqq \Sigma_{j}\left|\left([A-B] G_{j}^{\prime} U_{j}^{*} y, U_{j}^{*} z\right)\right|+\frac{1}{2}<1 .
\end{aligned}
$$

Thus $\alpha$ (and, similarly, $\alpha^{-1}$ ) is ultra-weakly continuous on $\mathfrak{A}$; and, from Lemma 3 , has an extension $\bar{\alpha}$ which is an automorphism of $\mathfrak{A}^{-}$satisfying $\|\bar{\alpha}-\iota\|=\|\alpha-\iota\|<2$. With $P$ a central projection in $\mathfrak{A}^{-}, \bar{\alpha}(P)=P$, since $\|\bar{\alpha}(P)-P\|=\frac{1}{2}\|\bar{\alpha}(2 P-I)-2 P+I\|<1$, and $\bar{\alpha}(P)$ and $P$ are commuting projections. (We can go on to show that $\bar{\alpha}$ is spatial, though we shall not use this fact. It is sufficient to prove that $\bar{\alpha}$ preserves the multiplicity function of $\mathfrak{H}^{-}$[15; Theorem 4.4.2], and since $\bar{\alpha}$ acts identically on the center it remains only to show that $\bar{\alpha}$ preserves maximal cyclicity of projections in countably decomposable central portions of $\mathfrak{A}$. Let $E$ be a projection in $\mathfrak{A}^{-}$which is maximal cyclic in $\mathfrak{A}-C_{E}$, where $C_{E}$ is the central carrier of $E$. With $F=\bar{\alpha}(E)$, the argument used above shows that $\|F-E\|<1$. Hence $\|F-F E F\|<1$, and the self-adjoint operator $F E F$ is one to one on the range of $F$, zero on its orthogonal complement, and so has range projection $F$. Thus $F E$ has range projection $F$; a similar argument shows that $E F\left(=(F E)^{*}\right)$ has range projection $E$, so $E \sim F$, and $F(=\bar{\alpha}(E))$ is maximal cyclic in $\left.\mathfrak{Q}-C_{E}\right)$. 
Lemma 5. Let $\alpha$ be an inner automorphism of a von Neumann algebra $\mathscr{R}$, for which $\|\alpha-\iota\|<2$. Then there is a unitary operator $U$ in $\mathscr{R}$, with spectrum $\sigma(U)$ in the half-plane $\left\{z: \operatorname{Re} z \geqq \frac{1}{2}\left(4-\|\alpha-\iota\|^{2}\right)^{\frac{1}{2}}\right\}$, such that $\alpha(A)=U A U^{*}$ for all $A$ in $\mathscr{R}$.

Proof. The argument is divided into three distinct stages. The first part proves the lemma when $\mathscr{R}$ is the algebra $\mathscr{M}_{n}$ of all operators on an $n$-dimensional Hilbert space, $n$ being an integer. This special case is used, in the second part, to obtain a weaker form of the lemma in which $\sigma(U)$ is contained in a slightly larger half-plane. Finally, the full lemma is deduced from this weaker form.

(a) We assume that $\mathscr{R}=\mathscr{M}_{n}$. Let $V$ be a unitary operator in $\mathscr{M}_{n}$ such that $\alpha(A)=V A V^{*}$ for each $A$ in $\mathscr{M}_{n}$, and let $a$ be the point in the convex hull of $\sigma(V)$ which is nearest to 0 . There are distinct points $a_{1}, \ldots, a_{q}$ in $\sigma(V)$, positive real numbers $c_{1}, \ldots, c_{q}$ with sum 1 such that $a=c_{1} a_{1}+\cdots+c_{q} a_{q}$, and unit vectors $x_{1}, \ldots, x_{q}$ such that $V x_{j}=a_{j} x_{j}$ $(j=1, \ldots, q)$. Since $x_{1}, \ldots, x_{q}$ are pairwise orthogonal, the unit vector $x=c_{1}^{\frac{1}{2}} x_{1}+\cdots+c_{q}^{\frac{1}{2}} x_{q}$ satisfies $(V x, x)=a$. Let $E$ and $F$ be the 1-dimensional projections with $x$ and $V x$, respectively, in their ranges. Then

$$
F=V E V^{*}=\alpha(E) \text {, so }
$$$$
\|\alpha-\iota\| \geqq\|\alpha(2 E-I)-2 E+I\|
$$$$
=2\|F-E\| \geqq 2\|V x-E V x\|=2\|V x-(V x, x) x\|=2\left(1-|a|^{2}\right)^{\frac{1}{2}} .
$$

Thus $|a| \geqq \frac{1}{2}\left(4-\|\alpha-\iota\|^{2}\right)^{\frac{1}{2}}>0$. With $U=(\bar{a}|| a \mid) V, U$ is a unitary element of $\mathscr{M}_{n}$ such that $\alpha(A)=U A U^{*}$ for all $A$ in $\mathscr{M}_{n}$, and $\sigma(U)$ lies in $\left\{z: \operatorname{Re} z \geqq \frac{1}{2}\left(4-\|\alpha-\iota\|^{2}\right)^{\frac{1}{2}}\right\}$.

(b) With $\mathscr{R}$ now a general von Neumann algebra, let $V$ be a unitary operator in $\mathscr{R}$ such that $\alpha(A)=V A V^{*}$ for each $A$ in $\mathscr{R}$. Choose a real number $k$ such that $0<k<\frac{1}{2}\left(4-\|\alpha-\iota\|^{2}\right)^{\frac{1}{2}}$. We shall show that $\alpha$ can be implemented by a unitary operator $U$ in $\mathscr{R}$ with $\sigma(U) \cong$ $\leqq\{z: \operatorname{Re} z \geqq k\}$. For each non-zero central projection $P$ in $\mathscr{R}$ let $d(P)$ denote the distance from 0 to the convex hull of the spectrum $\sigma_{P}(P V)$ of $P V$ (considered as a unitary operator on the range of $P$ ). We first prove that each such $P$ contains a non-zero central subprojection $Q$ such that $d(Q) \geqq k$. Suppose, to the contrary, that $P$ contains no such $Q$. Given $\varepsilon>0$ such that $\|\alpha-\imath\|+2 \varepsilon<2$, we can choose spectral projections $E_{1}, \ldots, E_{m}$ for $V$, with sum $I$, and complex numbers $a_{1}, \ldots, a_{m}$ of modulus 1 , such that $\|V-W\|<\varepsilon$, where $W=\sum_{j=1}^{m} a_{j} E_{j}$. With $\beta$ the automorphism of $\mathscr{R}$ defined by $\beta(A)=W A W^{*},\|\alpha-\beta\|<2 \varepsilon$ 
and so $\|\beta-\imath\|<\|\alpha-\imath\|+2 \varepsilon<2$. With $P_{j}$ the central carrier of $E_{j}$, and $Q$ the product of a maximal subset of $\left\{P, P_{1}, \ldots, P_{m}\right\}$ containing $P$ with non-null intersection, $Q$ is a non-zero central subprojection of $P$ for which each $Q E_{j}$ either is 0 or has central carrier $Q$. Renumber so that, for some $n \leqq m, Q E_{j}$ is non-zero if and only if $1 \leqq j \leqq n$.

By hypothesis, $d(Q)<k$, so we may choose $b_{1}, \ldots, b_{q}$ in $\sigma_{Q}(Q V)$ and positive real numbers $c_{1}, \ldots, c_{q}$ with sum 1 , such that $\left|c_{1} b_{1}+\cdots+c_{q} b_{q}\right|<k$. Since $\left\|Q V-\sum_{j=1}^{n} a_{j} Q E_{j}\right\|=\|Q(V-W)\|<\varepsilon$, each of $b_{1}, \ldots, b_{q}$ is at distance less than $\varepsilon$ from $\sigma_{Q}(Q W)=\left\{a_{1}, \ldots, a_{n}\right\}$. (Recall that, if $A$ and $B$ are normal operators and $\lambda \in \sigma(A)$, then the distance $d$ from $\lambda$ to $\sigma(B)$ is at most $\|A-B\|$. For by spectral theory, $d=\left\|(B-\lambda I)^{-1}\right\|^{-1}$; and if $d>\|A-B\|$ then $\|(A-\lambda I)-(B-\lambda I)\|$ $<\left\|(B-\lambda I)^{-1}\right\|^{-1}$, which implies [8; Lemma 1, p. 584] that $A-\lambda I$ has an inverse, contrary to hypothesis). Replacing $b$ 's by appropriate $a$ 's, we obtain a convex combination $a_{0}$ of $a_{1}, \ldots, a_{n}$ for which $\left|a_{0}\right|<k+\varepsilon$.

Let $F_{1}, \ldots, F_{n}$ be equivalent projections in $\mathscr{R}$ such that $0<F_{j} \leqq Q E_{j}$ $(1 \leqq j \leqq n)$, and choose partial isometries $F_{i j}(i, j=1, \ldots, n)$ in $\mathscr{R}$, with $F_{j j}=F_{j}$, which form a set of matrix units in a ${ }^{*}$-subalgebra $\mathscr{M}$ of $\mathscr{R}$ which is isomorphic to $\mathscr{M}_{n}$. With $W_{0}=\sum_{j=1}^{n} a_{j} F_{j}, W_{0}$ is unitary when considered as an element of $\mathscr{M}$, and $W_{0} F_{i j} W_{0}^{*}=a_{i} \bar{a}_{j} F_{i j}=W F_{i j} W^{*}$ $=\beta\left(F_{i j}\right)$. Hence the restriction $\gamma$ of $\beta$ to $\mathscr{M}$ is an automorphism of $\mathscr{M}$ which is implemented by $W_{0}$. Since $\|\gamma-\imath\| \leqq\|\beta-\imath\|<\|\alpha-\imath\|+2 \varepsilon<2$, while $a_{0}$ is a convex combination of $a_{1}, \ldots, a_{n}$ and so lies in the convex hull of the spectrum of $W_{0}$, we deduce from part (a) that

$$
\left|a_{0}\right| \geqq \frac{1}{2}\left[4-(\|\alpha-\iota\|+2 \varepsilon)^{2}\right]^{\frac{1}{2}} .
$$

This, with our previous estimate for $\left|a_{0}\right|$, gives

$$
k>\frac{1}{2}\left[4-(\|\alpha-\iota\|+2 \varepsilon)^{2}\right]^{\frac{1}{2}}-\varepsilon,
$$

contradicting the assumption that

with suitably chosen $\varepsilon$.

$$
k<\frac{1}{2}\left(4-\|\alpha-\iota\|^{2}\right)^{\frac{1}{2}}
$$

We have now shown that each non-zero central projection $P$ in $\mathscr{R}$ contains a non-zero central subprojection $Q$ for which $d(Q) \geqq k$. It follows that there is an orthogonal family $\left\{Q_{j}\right\}$ of central projections, with sum $I$, such that $d\left(Q_{j}\right) \geqq k$. With $a_{j}$ the point in the convex hull of $\sigma_{Q_{j}}\left(Q_{j} V\right)$ which is closest to $0,\left|a_{j}\right| \geqq k>0$ and $\left(\bar{a}_{j}|| a_{j} \mid\right) Q_{j} V$ has spectrum in $\{z: \operatorname{Re} z \geqq k\}$. Hence $U=\left(\Sigma\left(\bar{a}_{j} /\left|a_{j}\right|\right) Q_{j}\right) V$ is a unitary operator 
in $\mathscr{R}$, with spectrum in the same half-plane, such that $\alpha(A)=U A U^{*}$ for each $A$ in $\mathscr{R}$.

(c) For $c$ in $\left[0, \frac{1}{2} \pi\right)$ define $S_{c}=\{\exp i t:-c \leqq t \leqq c\}$, so that $S_{c}$ is the are of the unit circle that lies in the half-plane $\{z: \operatorname{Re} z \geqq \cos c\}$. We can choose $b$ in $\left[0, \frac{1}{2} \pi\right)$ so that $\|\alpha-\imath\|=2 \sin b$, whence

$$
\cos b=\left(4-\|\alpha-\iota\|^{2}\right)^{\frac{1}{2}}
$$

and we have to show that $\alpha$ can be implemented by a unitary operator $U$ in $\mathscr{R}$ with $\sigma(U) \leqq S_{b}$.

Choose real numbers $c, \delta$ such that $b<c<\frac{1}{2} \pi$ and $0<\delta<\frac{1}{2} \cos c$, and let $\varepsilon_{n}=(c-b)(1-\delta)^{n-1}(n=1,2, \ldots)$. We shall construct inductively a sequence $\left\{U_{n}\right\}$ of unitary operators in $\mathscr{R}$, each of which implements $\alpha$, such that

$$
\sigma\left(U_{n}\right) \leqq S_{b+\varepsilon_{n}}, \quad\left\|U_{n+1}-U_{n}\right\| \leqq\left|1-\exp \left(i \delta \varepsilon_{n}\right)\right| .
$$

Since $0<\cos c<\cos b=\frac{1}{2}\left(4-\|\alpha-\iota\|^{2}\right)^{\frac{1}{2}}$, it follows from part (b), with $k=\cos c$, that there is a unitary operator $U_{1}$ in $\mathscr{R}$ which implements $\alpha$ and has $\sigma\left(U_{1}\right)$ a subset of $S_{c}=S_{b+\varepsilon_{1}}$. Suppose that a unitary operator $U_{n}$ in $\mathscr{R}$ has been constructed, with $U_{n}$ implementing $\alpha$ and $\sigma\left(U_{n}\right) \leqq S_{b+\varepsilon_{n}}$. Let $E$ and $F$ be the spectral projections for $U_{n}$ corresponding to the Borel sets

and

$$
\left\{\exp i t: b+(1-2 \delta) \varepsilon_{n} \leqq t \leqq b+\varepsilon_{n}\right\}
$$

$$
\left\{\exp -i t: b+(1-2 \delta) \varepsilon_{n} \leqq t \leqq b+\varepsilon_{n}\right\},
$$

respectively. Suppose that $\mathscr{R}$ contains a non-zero partial isometry $W$ with initial and final projections dominated by $E$ and $F$, respectively. Then

and

$$
\begin{aligned}
& \left\|E U_{n}-\exp \left(i b+i \varepsilon_{n}\right) E\right\|<2 \delta \varepsilon_{n}, \\
& \left\|U_{n} F-\exp \left(-i b-i \varepsilon_{n}\right) F\right\|<2 \delta \varepsilon_{n}
\end{aligned}
$$

Thus

whence

$$
W E=F W=W \text {. }
$$

$$
\begin{array}{rl}
\|\alpha-\iota\| \geqq \| W U_{n}-U_{n} & W \| \\
& \geqq\left|\exp \left(i b+i \varepsilon_{n}\right)-\exp \left(-i b-i \varepsilon_{n}\right)\right|\|W\|-4 \delta \varepsilon_{n} \\
& =2 \sin \left(b+\varepsilon_{n}\right)-4 \delta \varepsilon_{n} \\
& =2 \sin b+2\left\{\sin \left(b+\varepsilon_{n}\right)-\sin b\right\}-4 \delta \varepsilon_{n} \\
& >2 \sin b+2(\cos c) \varepsilon_{n}-4 \delta \varepsilon_{n} \\
& >2 \sin b=\|\alpha-\imath\|,
\end{array}
$$


a contradiction. Hence no such $W$ exists, and there is a central projection $Q$ in $\mathscr{R}$ such that $E \leqq Q$ and $F \leqq I-Q$. Thus $\sigma_{Q}\left(Q U_{n}\right)$ and $\sigma_{I-Q}\left((I-Q) U_{n}\right)$ are contained in the arcs

and

$$
\left\{\exp i t:-b-(1-2 \delta) \varepsilon_{n} \leqq t \leqq b+\varepsilon_{n}\right\}
$$

$$
\left\{\exp i t:-b-\varepsilon_{n} \leqq t \leqq b+(1-2 \delta) \varepsilon_{n}\right\},
$$

respectively. Since $(1-\delta) \varepsilon_{n}=\varepsilon_{n+1}$, the unitary operator

$$
U_{n+1}=\left\{\exp \left(-i \delta \varepsilon_{n}\right) Q+\exp \left(i \delta \varepsilon_{n}\right)(I-Q)\right\} U_{n}
$$

has spectrum in $S_{b+\varepsilon_{n+1}}$. It is clear that $U_{n+1}$ implements $\alpha$ and satisfies (**). This completes the inductive construction of the sequence $\left\{U_{n}\right\}$.

Since $\Sigma \varepsilon_{n}<\infty,\left\{U_{n}\right\}$ converges in the norm topology to a unitary operator $U$ in $\mathscr{R}$ which implements $\alpha$. Each point of $\sigma(U)$ is at distance at most $\left\|U-U_{n}\right\|$ from $\sigma\left(U_{n}\right)$ (as noted, in part (b), for any two normal operators), and since $\sigma\left(U_{n}\right) \leqq S_{b+\varepsilon_{n}}$ and $\left\|U-U_{n}\right\| \rightarrow 0$, it follows that $\sigma(U) \leqq S_{b}$.

Remark $A$. The condition on the spectrum of $U$ established in Lemma 5 can be reinterpreted more geometrically as saying that $\sigma(U)$ lies on the arc of the unit circle symmetric about 1 with endpoints midway between 1 and the points at distance $\|\alpha-\iota\|$ from 1 . Having proved this under the assumption $\|\alpha-\imath\|<2$, our operator $U$ is chosen with spectrum in the "open right half-plane" $(\operatorname{Re} z>0)$.

Remark $B$. Let $U$ be a unitary operator on a Hilbert space $\mathscr{H}$, for which the convex hull of $\sigma(U)$ contains a neighbourhood of 0 , and let $\alpha$ be the (inner) automorphism induced by $U$ on $\mathscr{B}(\mathscr{H})$. Every other unitary operator in $\mathscr{B}(\mathscr{H})$ which implements $\alpha$ is a multiple of $U$ by a complex number of modulus 1 ; and no such multiple has spectrum in the right half-plane. It follows from Lemma 5 that $\|\alpha-\imath\|=2$ (a fact that can easily be proved directly by reasoning as in part (a) of the proof of Lemma 5, after approximating $U$ by a unitary operator $V$ which is a finite linear combination of spectral projections for $U$ ). This example shows that the conclusion of Lemma 5 can fail to hold when $\|\alpha-\imath\|=2$.

If we restrict $U$ further by requiring in addition that $U^{3}=I$, then $\alpha^{3}=\iota$, and the spectrum of $\alpha$ as an operator on $\mathscr{B}(\mathscr{H})$ consists of third roots of unity. Thus $\alpha-\iota$ has spectral radius $r$ at most $\sqrt{3}$. It follows that the statement obtained from Lemma 5, upon replacing $\|\alpha-\imath\|$ throughout by $r$, is false. It should be noted that the spectrum of $\alpha$ is a subset of $\left\{a b^{-1}: a, b\right.$ in $\left.\sigma(U)\right\}$, which is consistent with the possibility of choosing $U$ with $\sigma(U)$ in the closed right half-plane $\left\{z: \operatorname{Re} z \geqq \frac{1}{2}\left(4-r^{2}\right)^{\frac{1}{2}}\right\}$.

Lemma 6. If $\mathfrak{A}$ is a $C^{*}$-algebra and $U$ a unitary operator acting on a Hilbert space $\mathscr{H}$ such that $\alpha(A)=U A U^{*}$ lies in $\mathfrak{A}$ for all $A$ in $\mathfrak{A}$ and 
$\operatorname{Re} a>0$ for each $a$ in $\sigma(U)$, then $\alpha$ lies on a norm-continuous one-parameter subgroup of $\alpha(\mathfrak{U})$ and is $\pi$-inner.

Proof. By hypothesis on $\sigma(U)$, we can choose $H$ self-adjoint with $\sigma(H)$ in $(-\pi / 2, \pi / 2) \operatorname{such}$ that $U=\exp i H$. As in Lemma $2, \bar{\alpha}=\exp (\operatorname{ad} i H)$ both as a power series and as an analytic function of the bounded linear operator ad $i H$ acting on the Banach space $\mathscr{B}(\mathscr{H})$, where $\bar{\alpha}$ is the extension of $\alpha$ to $\mathscr{B}(\mathscr{H})$ defined by $\bar{\alpha}(B)=U B U^{*}$. From Gardner [9: Corollary 3], taking $\mathscr{B}(\mathscr{H})$ as the Banach algebra and $\mathscr{A}$ as the invariant subspace of that statement, we have that $\mathfrak{A}$ is invariant under $A \rightarrow \exp (i s H) A \exp (-i s H)$ for all real $s$, since there is no difficulty in identifying $i H$ as $\log U$ in the sense GARDNER uses for " $\log U$ ". Thus $\bar{\alpha}$ lies on a norm-continuous one-parameter subgroup of $\alpha(\mathfrak{A})$. It seems worthwhile to include our original proof of this both for completeness and directness.

With $T$ in $\mathscr{B}(\mathscr{H})$, we denote by $L(T)$ and $R(T)$ the (bounded) operators on $\mathscr{B}(\mathscr{H})$ defined by $L(T)(A)=T A$ and $R(T)(A)=A T$. Since $L$ and $R$ are algebraic isomorphism and anti-isomorphism of $\mathscr{B}(\mathscr{H})$ into the Banach algebra $\mathscr{B}(\mathscr{B}(\mathscr{H}))$ of bounded operators on $\mathscr{B}(\mathscr{H})$ each of which maps $I$ onto $\iota$ the spectra of $L(T)$ and $R(T)$ are contained in the spectrum of $T$. Let $\mathscr{A}$ be a maximal commutative subalgebra of $\mathscr{B}(\mathscr{B}(\mathscr{H}))$ containing $L(T)$ and $R(T)$. By maximality an element $\beta$ of $\mathscr{A}$ has an inverse in $\mathscr{B}(\mathscr{B}(\mathscr{H}))$ if and only if it has an inverse in $\mathscr{A}$, so that the spectra of $\beta$ relative to $\mathscr{A}$ and $\mathscr{B}(\mathscr{B}(\mathscr{H})$ ) coincide. Since each element of the spectrum of $\beta$ is the image of $\beta$ under a multiplicative linear functional on $\mathscr{A}$, the spectrum of $L(T)-R(T)(=\operatorname{ad} T)$ is contained in $\{a-b$ : $a, b$ in the spectrum of $T\}$. In particular ad $i H$ has spectrum in $\{i t:|t| \leqq r\}$, where $2\|H\|=r<\pi$, by choice of $H$. From $\bar{\alpha}=\exp (\operatorname{ad} i H)$ and the spectral mapping theorem [8; VII. 3.11], $\bar{\alpha}$ has spectrum in $\{\exp i t:|t| \leqq r\}$.

For each real $s$, let $g_{s}$ denote the principal value of $z \rightarrow z^{s}$ on the plane of complex numbers slit along the negative axis; and $\tilde{s}$, multiplication by $s$. On the $\operatorname{strip} S=\{z:|\operatorname{Im} z|<\pi\}$ we have $g_{s} \circ \exp =\exp \circ \tilde{s}$. Since $g_{s}$, exp and $\tilde{s}$ are analytic where defined and ad $i H$ has spectrum in $S$, $\bar{\alpha}^{s}\left(=g_{s}(\bar{\alpha})\right)=\exp (s \operatorname{ad} i H)=\exp (\operatorname{ad} i s H)$, for all real $s$, from [8; VII. 3.12]. Since $\operatorname{ad}(i s H)$ is a derivation of $\mathscr{B}(\mathscr{H})$ and $i s H$ is skewadjoint, $\bar{\alpha}^{s}$ is an automorphism of $\mathscr{B}(\mathscr{H})$ (cf. Lemma 2 ).

Having identified the spectrum of $\bar{\alpha}$ as a subset of $\{\exp i t:|t| \leqq r<\pi\}$ $\left(=C_{0}\right)$, we can choose a compact set $K$ with $C_{0}$ in its interior $K_{0}$ and a rectifiable Jordan curve $C$ in the plane slit along the negative axis having $K$ in its interior such that $z \rightarrow\left(z_{0}-z\right)^{-1}$ is uniformly approximable on $K$ by polynomials in $z$ (from Runge's theorem) for each $z_{0}$ on $C$. Then $\left(z_{0}-\bar{\alpha}\right)^{-1}$ is a uniform limit of polynomials in $\bar{\alpha}$, from [8; VII. 3.13], 
for each $z_{0}$ on $C$; so that $\left(z_{0}-\bar{\alpha}\right)^{-1}$ leaves $\mathfrak{A}$ invariant. Now,

$$
\bar{\alpha}^{s}=\frac{1}{2 \pi i} \int_{C} g_{s}(z)(z-\bar{\alpha})^{-1} d z ;
$$

so that $\bar{\alpha}^{s}$ leaves $\mathfrak{A}$ invariant [8, VII. 3.9]. Again, $g_{s}$ converges uniformly to the constant function 1 on $K_{0}$ as $s \rightarrow 0$; so that $\left\|\bar{\alpha}^{s}-\imath\right\| \rightarrow 0$ as $s \rightarrow 0$ from [8; VII. 3. 13]. Finally, $\bar{\alpha}^{s} \bar{\alpha}^{t}=\bar{\alpha}^{s+t}$ from [8; VII. 3.10(b)], since $g_{s} \cdot g_{t}=g_{s+t}$; so that $s \rightarrow \bar{\alpha}^{s} \mid \mathfrak{A}$ is a norm-continuous, one-parameter subgroup of $\alpha(\mathfrak{I})$ with $\alpha=\bar{\alpha}^{1} \mid \mathfrak{A}$.

Remark $C$. With $\mathfrak{M}$ the (factor) group algebra of the free group on two generators $a$ and $b$, the automorphism of the group arising from interchanging $a$ and $b$ gives rise to an outer automorphism $\alpha$ of $\mathfrak{M}[6$; Exercise 15, p. 308] and a unitary operator $U$ implementing it. Since $U$ is of order two, its spectrum consists of -1 and 1 ; so that $i U$ has spectrum in the closed right half-plane, and implements $\alpha$. Thus the conclusion of Lemma 6 above need not hold if the hypothesis is weakened to allow $\sigma(U)$ to lie in the closed right half-plane.

It is now a simple matter to assemble the preceding lemmas in our main result.

Theorem 7. If $\alpha$ is an automorphism of a $C^{*}$-algebra $\mathfrak{A}$ and $\|\alpha-\imath\|<2$, then $\alpha$ lies on a norm-continuous one-parameter subgroup of $\alpha(\mathfrak{A})$. Such subgroups generate $\gamma(\mathfrak{A})$, the connected component of $\iota$ in $\alpha(\mathfrak{A})$ with its norm topology, as a group; and $\gamma(\mathfrak{I})$ is an open subgroup of $\alpha(\mathfrak{I})$. Each element of $\gamma(\mathfrak{A})$ is $\pi$-inner.

Proof. Pass to the reduced atomic representation of $\mathfrak{A}$. We assume that $\mathfrak{A}$ acting on $\mathscr{H}$ is this (faithful) representation of $\mathfrak{A}$; so that $\mathfrak{A}^{-}$is of type $I$ - in fact, a direct sum of algebras of the form $\mathscr{B}\left(\mathscr{H}_{0}\right)[12$ : Corollary 4]. From Lemma 4 , there is an automorphism $\bar{\alpha}$ of $\mathfrak{Q}-$ leaving each element of the center of $\mathfrak{A}$ - fixed whose restriction to $\mathfrak{A}$ is $\alpha$. From [6; Corollary, p. 256], there is a unitary operator $U$ in $\mathfrak{A}$-implementing $\bar{\alpha}$; and from Lemma $5 U$ can be chosen with $\sigma(U)$ in the half-plane $\{a: \operatorname{Re} a>0\}$. Lemma 6 now tells us that $\alpha$ lies on a norm-continuous one-parameter subgroup of $\alpha(\mathfrak{U})$. Each such subgroup is a (norm) connected subset of $\alpha(\mathfrak{A})$ containing $\iota$, and, therefore, lies in $\gamma(\mathfrak{A})$ - as does the subgroup they generate. However this subgroup contains the interior of the ball of radius 2 about $\iota$ in $\alpha(\mathfrak{U})$ (as we have just shown); so that it is open in $\alpha(\mathfrak{A})$, hence, closed, and no larger subset of $\alpha(\mathfrak{A})$ is connected. Thus this subgroup coincides with $\gamma(\mathfrak{U})$. Since the normcontinuous one-parameter subgroups of $\alpha(\mathfrak{A})$ consist of $\pi$-inner automorphisms of $\mathfrak{A}$ (Lemma 2), each element of $\gamma(\mathfrak{Z})$ is $\pi$-inner.

Remark $D$. Note that, after passing to the reduced atomic representation and by restricting to a minimal central projection, it is necessary to employ Lemma 5 only in the case where $\mathscr{R}$ is $\mathscr{B}(\mathscr{H})$ for the proof of 
Theorem 7. After approximation by spectral theory, the proof of Lemma 5 , in case $\mathscr{R}=\mathscr{B}(\mathscr{H})$ is essentially part (a) of the proof given.

Remark $E$. Applying Theorem 7 and then Lemma 5, we see that Lemma 5 holds with "inner" deleted, $\mathscr{R}$ a $C^{*}$-algebra and " $\mathscr{R}$ "' replacing the second occurrence of " $\mathscr{R}$ ".

Remark $F$. The following example shows that the statement obtained from the first sentence of Theorem 7, upon replacing $\|\alpha-\imath\|$ by the spectral radius of $\alpha-\imath$, is false even for von Neumann algebras.

With $\mathfrak{M}$ the (factor) group algebra of the free group on three generators $a, b, c$, permuting these generators cyclically induces an automorphism $\alpha$ of $\mathfrak{M}$ and a unitary operator $U$ (of order three) with $\sigma(U)$ the third roots of unity and implementing $\alpha$. By the reasoning used at the end of Remark $B, \alpha-\iota$ has spectral radius at most $\sqrt{3}$. A slight extension of [6; Exercise 15, p. 308] shows that $\alpha$ is an outer automorphism. Since $\mathfrak{M}$ is weakly closed, $\alpha$ is not $\pi$-inner, hence (Lemmas 2 and 6 ) does not lie on a norm-continuous one-parameter subgroup of $\alpha(\mathfrak{W})$ and cannot be implemented by a unitary operator having spectrum in the open right half-plane. It follows from Theorem 7 that $\|\alpha-\imath\|=2$ (a fact that can be verified directly: for $\left\|(\alpha-\iota)\left(U_{a}\right)\right\|=\left\|U_{a}-U_{b}\right\|$ $=\left\|U_{b^{-1} a}-I\right\|=2$, since $U_{b^{-1} a}$ leaves the space of functions square summable on the group and vanishing on positive powers of $a^{-1} b$ invariant, while $U_{b^{-1} a}^{*}$ does not; so that $\sigma\left(U_{b^{-1} a}\right)$ is the entire unit circle and, in particular, -2 is in $\left.\sigma\left(U_{b^{-1} a}-I\right)\right)$.

Since each norm- continuous representation of a connected topological group by automorphisms of a $C^{*}$-algebra $\mathfrak{A}$ has range in $\gamma(\mathfrak{A})$, we have:

Corollary 8. Each norm-continuous representation of a connected topological group by automorphisms of a $C^{*}$-algebra has range consisting of $\pi$-inner automorphisms.

In the case of von Neumann algebras, we have:

Corollary 9. If $\mathfrak{A}$ is a $C^{*}$-algebra which has a faithful representation $\varphi$ as a von Neumann algebra then $\iota_{0}(\mathfrak{U})=\gamma(\mathfrak{A})=\pi(\mathfrak{U})=\iota_{\varphi}(\mathfrak{A})$; and each element of $\gamma(\mathfrak{U})$ lies on some norm-continuous one-parameter subgroup of $\alpha(\mathfrak{U})$.

Remark $G$. Let $\mathfrak{A}$ be a $C^{*}$-algebra, $\varphi$ a faithful representation of $\mathfrak{A}$. It follows at once from Definition that $\pi(\mathfrak{U}) \leqq \iota_{\varphi}(\mathfrak{A}) \leqq \sigma_{\varphi}(\mathfrak{A}) \leqq \varepsilon_{\varphi}(\mathfrak{U}) \leqq$ $\leqq \alpha(\mathfrak{U})$. Theorem 7 provides the additional information that $\gamma(\mathfrak{Z}) \leqq$ $\leqq \pi(\mathfrak{Z})$, and hence that each of the groups listed above contains the open ball, with center $\iota$ and radius 2 , in $\alpha(\mathfrak{U})$. It follows that each of these groups is open, hence closed, and that the quotient of any one of them by a smaller one is discrete.

The subgroups $\gamma(\mathfrak{U})$ and $\pi(\mathfrak{U})$ of $\alpha(\mathfrak{A})$ are normal. For suppose that $\alpha \in \alpha(\mathfrak{U})$ and $\beta \in \pi(\mathfrak{U})$. Given any faithful representation $\psi$ of $\mathfrak{A}$, $\psi\left(\alpha \beta \alpha^{-1}\right) \psi^{-1}=(\psi \alpha) \beta(\psi \alpha)^{-1}$ and, since $\psi \alpha$ is a faithful representation of $\mathfrak{A}, \psi\left(\alpha \beta \alpha^{-1}\right) \psi^{-1}$ is a weakly-inner automorphism of $(\psi \alpha)(\mathfrak{U})=\psi(\mathfrak{U})$. 
Hence $\alpha \beta \alpha^{-1} \in \pi(\mathfrak{U})$, so $\pi(\mathfrak{U})$ is a normal subgroup of $\alpha(\mathfrak{U})$; the same is true of $\gamma(\mathfrak{U})$ since it is the connected component of the identity in $\alpha(\mathfrak{U})$.

We now exhibit a $C^{*}$-algebra $\mathfrak{A}$ and a faithful representation $\varphi$ of $\mathfrak{A}$ for which the subgroups $\iota_{\varphi}(\mathfrak{U}), \sigma_{\varphi}(\mathfrak{U})$ and $\varepsilon_{\varphi}(\mathfrak{U})$ of $\alpha(\mathfrak{U})$ are not normal. For this purpose we make use of Example $a$, in which an automorphism $\beta$ of a $C^{*}$-algebra $\mathscr{B}$ is produced, as well as faithful representations $\psi$ and $\theta$ of $\mathscr{B}$ for which $\psi \beta \psi^{-1}$ is weakly-inner while $\theta \beta \theta^{-1}$ is not extendable. Let $\mathfrak{A}$ be $\mathscr{B} \oplus \mathscr{B}, \varphi$ the faithful representation $\left(B_{1}, B_{2}\right) \rightarrow\left(\psi\left(B_{1}\right), \theta\left(B_{2}\right)\right)$ of $\mathfrak{A}, \alpha$ and $\gamma$ the automorphisms of $\mathfrak{A}$ for which $\alpha\left(\left(B_{1}, B_{2}\right)\right)=\left(\beta\left(B_{1}\right), B_{2}\right)$, $\gamma\left(\left(B_{1}, B_{2}\right)\right)=\left(B_{2}, B_{1}\right)$. Then $\varphi(\mathfrak{A})=\psi(\mathscr{B}) \oplus \theta(\mathscr{B})$, and since $\left(\gamma \propto \gamma^{-1}\right)\left(\left(B_{1}, B_{2}\right)\right)=\left(B_{1}, \beta\left(B_{2}\right)\right)$, it is readily verified that $\varphi \alpha \varphi^{-1}$ is weakly-inner, while $\varphi\left(\gamma \propto \gamma^{-1}\right) \varphi^{-1}$ is not extendable. Thus $\alpha \in \iota_{\varphi}(\mathfrak{A})$, $\gamma \propto \gamma^{-1} \notin \varepsilon_{\varphi}(\mathfrak{U})$, whence the subgroups $\iota_{\varphi}(\mathfrak{U}), \sigma_{\varphi}(\mathfrak{A})$ and $\varepsilon_{\varphi}(\mathfrak{U})$ of $\alpha(\mathfrak{A})$ are not normal.

For each $C^{*}$-algebra $\mathfrak{A}$ the subgroup $\iota_{\varphi}(\mathfrak{U})$ of $\varepsilon_{\varphi}(\mathfrak{A})$ is normal. With $\alpha$ in $\iota_{\varphi}(\mathfrak{A}), \beta$ in $\varepsilon_{\varphi}(\mathfrak{A}), U$ a unitary operator in $\varphi(\mathfrak{A})^{-}$which implements $\varphi \propto \varphi^{-1}$ and $\gamma$ an automorphism of $\varphi(\mathfrak{A})^{-}$which extends $\varphi \beta \varphi^{-1}, \gamma(U)$ is a unitary operator in $\varphi(\mathfrak{Z})^{-}$which implements $\varphi\left(\beta \alpha \beta^{-1}\right) \varphi^{-1}$. Thus $\beta \alpha \beta^{-1} \in \iota_{\varphi}(\mathfrak{U})$ and $\iota_{\varphi}(\mathfrak{U})$ is a normal subgroup of $\varepsilon_{\varphi}(\mathfrak{U})$.

We now give an example in which $\mathfrak{A}$ is an abelian $C^{*}$-algebra with a faithful representation $\varphi(\mathfrak{U})$ acting on a finite-dimensional Hilbert space, and the subgroup $\sigma_{\varphi}(\mathfrak{A})$ of $\varepsilon_{\varphi}(\mathfrak{U})$ is not normal (of course, $\varepsilon_{\varphi}(\mathfrak{U})$ $=\alpha(\mathfrak{A})$ in this case, since $\varphi(\mathfrak{A})$ is finite-dimensional and so weakly closed). Let $\mathfrak{A}$ be the algebra of all complex $4 \times 4$ diagonal matrices of the form $\operatorname{diag}(a, a, b, c), \varphi$ a representation in which $\mathfrak{A}$ acts in the obvious way on a 4-dimensional Hilbert space. With $\alpha$ (respectively, $\sigma$ ) the automorphism of $\mathfrak{A}$ corresponding to interchange of $a$ and $b$ (respectively, $b$ and $c$ ), it is clear that $\sigma \in \sigma_{\varphi}(\mathfrak{U})$. However, $\alpha \sigma \alpha^{-1}$ is the automorphism of $\mathfrak{A}$ corresponding to interchange of $\mathrm{a}$ and $\mathrm{c}$, and consideration of the multiplicities of the eigenvalues of $A$ (in $\mathfrak{A}$ ) and of $\left(\alpha \sigma \alpha^{-1}\right)(A)$ shows that $\alpha \sigma \alpha^{-1} \notin \sigma_{\varphi}(\mathfrak{U})$.

The group $\iota_{0}(\mathfrak{U})$ of inner automorphisms of a general $C^{*}$-algebra $\mathfrak{A}$ is contained in $\pi(\mathfrak{U})$, and is a normal subgroup of $\alpha(\mathfrak{U})$. For if $\beta$ is the inner automorphism implemented by a unitary element $U$ of $\mathfrak{A}$, and $\alpha \in \alpha(\mathfrak{A})$, then $\alpha \beta \alpha^{-1}$ is the inner automorphism induced by $\alpha(U)$.

Suppose now that $\mathfrak{A}$ is a $C^{*}$-algebra having a faithful representation $\varphi$ for which $\varphi(\mathfrak{A})$ is weakly closed. By Corollary $9, \gamma(\mathfrak{U})=\pi(\mathfrak{A})=\iota_{\varphi}(\mathfrak{A})$ $=\iota_{0}(\mathfrak{U})$, and of course $\varepsilon_{\varphi}(\mathfrak{U})=\alpha(\mathfrak{U})$. Hence there are now only three (possibly) distinct groups under consideration, and $\iota_{0}(\mathfrak{U}) \leqq \sigma_{\varphi}(\mathfrak{U}) \leqq \alpha(\mathfrak{U})$. We have already noted that $\iota_{0}(\mathfrak{A})$ is a normal subgroup of $\alpha(\mathfrak{A})$, and the finite dimensional example described above shows that the subgroup $\sigma_{\varphi}(\mathfrak{A})$ of $\alpha(\mathfrak{U})$ is not necessarily normal.

4 Commun. math. Phys., Vol. 4 


\section{Special cases}

In this section, we illustrate by example that various possibilities not ruled out by the results of $\S 3$ do occur. Notably, in the example which follows, we locate an automorphism weakly-inner in one faithful representation and not extendable in another - completing the normality discussion of Remark G. Some examples taking advantage of special properties of the ideal of compact operators follow this; and examples, making use of the detailed knowledge of the higher connectivity properties of certain compact spaces to allow us to compute, specifically, some automorphism subgroups, conclude this section.

Example $a$. We use the fermion algebra (cf. [27] and [11]) to establish that $\gamma(\mathfrak{A})$ need not coincide with $\iota_{\varphi}(\mathfrak{A})$ for some faithful representation $\varphi$ of $\mathfrak{A}$. Our algebra $\mathfrak{A}$ is characterised as a $C^{*}$-algebra by having a dense self-adjoint subalgebra which is the union of an ascending sequence of self-adjoint subalgebras $\mathscr{M}_{n}, n=1,2, \ldots$ each isomorphic to the algebra of complex $2^{n} \times 2^{n}$ matrices and all having the same unit. We shall exhibit an automorphism of $\mathfrak{A}$ and two faithful representations of $\mathfrak{A}$, in one of which the automorphism is weakly-inner and in the other of which it is not - indeed in which it is not extendable. Both representations are irreducible. It follows that this automorphism is not in the connected component of the identity $\gamma(\mathfrak{Q})$ in $\alpha(\mathfrak{Q})$, since each element of $\gamma(\mathfrak{Q})$ is weakly-inner in all faithful representations. For this purpose, we choose matrix units $\left\{E_{j k}^{(n)}\right\}, j, k=1, \ldots, 2^{n}$ in $\mathscr{M}_{n}$, with $E_{j j}^{(n)}, j=1, \ldots, 2^{n}$, orthogonal projections and $E_{j k}^{(n) *}=E_{k j}^{(n)}$, such that

$$
\begin{gathered}
E_{j j}^{(n-1)}=E_{2 j-12 j-1}^{(n)}+E_{2 j 2 j}^{(n)}, \\
E_{2 j-11}^{(n)}=E_{j 1}^{(n-1)} E_{11}^{(n)}, \\
E_{2 j 2}^{(n)}=E_{j 1}^{(n-1)} E_{22}^{(n)},
\end{gathered}
$$

for $n=1,2, \ldots$ and $j=1, \ldots, 2^{n-1}\left(\mathscr{M}_{0}\right.$ is the algebra of scalars and $E_{11}^{(0)}$ is $I$ ).

Let $\alpha$ be the automorphism of $\mathfrak{U}$ which on each $\mathscr{M}_{n}$ transposes a matrix about each diagonal, i.e. $\alpha\left(E_{j k}^{(n)}\right)=E_{2^{n}-j+12^{n}-k+1}^{(n)}$, so that $\alpha$ is the automorphism induced by the permutation matrix $U_{n}$ with entry $I$ at each position on the secondary diagonal. Since $U_{n+1}$ (in $\mathscr{M}_{n+1}$ ) induces the same automorphism on $\mathscr{M}_{n}$, there is an automorphism on the union of the $\mathscr{M}_{n}$ 's defined by this process. Since the automorphism on each $\mathscr{M}_{n}$ is isometric it has a unique extension to $\mathfrak{A}$ which is the desired automorphism $\alpha$. For our first representation, let $\mathscr{H}$ be $\mathscr{L}_{2}(0,1)$ relative to Lebesgue measure and let $E_{j k}^{(n)}$ be the isometric mapping of functions in $\mathscr{L}_{2}(0,1)$ vanishing outside of $\left[\frac{k-1}{2^{n}}, \frac{k}{2^{n}}\right]$ to those vanishing outside $\left[\frac{j-1}{2^{n}}, \frac{j}{2^{n}}\right]$ induced by translating the second interval onto the first (and, 
of course, consistent with $E_{j k}^{(n)}$ being a partial isometry, let it map functions vanishing on $\left[\frac{k-1}{2^{n}}, \frac{k}{2^{n}}\right]$ into 0$)$. In particular $E_{j j}^{(n)}$ is the operator which multiplies functions in $\mathscr{L}_{2}(0,1)$ by the characteristic function of $\left[\frac{j-1}{2^{n}}, \frac{j}{2^{n}}\right]$. Since each continuous function on $[0,1]$ is a uniform limit of finite linear combinations of such characteristic functions, the operators which are multiplication by such functions lie in $\mathfrak{A}$. An operator commuting with $\mathfrak{A}$ commutes with multiplications by continuous functions hence with multiplications by all bounded measurable functions and is, therefore, itself multiplication by a bounded measurable function (such multiplications forming a maximal abelian algebra). With $M_{f}$ multiplication by $f, M_{f} E_{j k}^{(n)}=E_{j k}^{(n)} M_{f}$ if and only if $f$ is invariant under the mapping which translates $\left[\frac{k-1}{2^{n}}, \frac{k}{2^{n}}\right]$ onto $\left[\frac{j-1}{2^{n}}, \frac{j}{2^{n}}\right]$, translates

$$
\left[\frac{j-1}{2^{n}}, \frac{j}{2^{n}}\right]
$$

onto $\left[\frac{k-1}{2^{n}}, \frac{k}{2^{n}}\right]$ and leaves the other points of $[0,1]$ fixed. For this to hold for all $j, k, n, f$ must be almost constant and $M_{f}$ a scalar. In fact, denoting by $U_{j k}^{(n)}$ the unitary operator $E_{j k}^{(n)}+E_{k j}^{(n)}+I-E_{j j}^{(n)}-E_{k k}^{(n)}, \quad U_{j k}^{(n)} f=f$ (note that $f$ is also in $\mathscr{L}_{2}(0,1)$ ). Let $g_{m}$ be a continuous function on $[0,1]$ with $\left\|f-g_{m}\right\|<\frac{1}{m}($ in $\mathscr{H})$ and choose $n$ such that if $\left|p-p^{\prime}\right|<1 / 2^{n}$ then $\left|g_{m}(p)-g_{m}\left(p^{\prime}\right)\right|<\frac{1}{m}$. Each permutation $\tau$ of the intervals $\left[\frac{j-1}{2^{n}}-\frac{j}{2^{n}}\right]$, $j=1, \ldots, 2^{n}$ corresponds to a unitary operator $U_{\tau}$ which is a product of the $U_{j k}^{(n)}\left(\right.$ these correspond to a transposition of $\left[\frac{j-1}{2^{n}}, \frac{j}{2^{n}}\right]$ and $\left.\left[\frac{k-1}{2^{n}}, \frac{k}{2^{n}}\right]\right)$; so that $U_{\tau} f=f$. With $S$ the (symmetric) group of all such permutations and $A$ the operator $\frac{1}{2^{n} !} \sum_{\tau \text { in } S} U_{\tau},\|A\| \leqq 1$ and $A f=f$. Thus $\left\|f-A g_{m}\right\|<\frac{1}{m}$. Since $U_{\tau} A g_{m}=A g_{m}$ and the oscillation of $A g_{m}$ over each interval $\left[\frac{j-1}{2^{n}}, \frac{j}{2^{n}}\right]$ is not greater than that of $g_{m}$ over such intervals, $A g_{m}$ differs from some constant $C_{m}$ by at most $\frac{1}{m}$ at each point of $[0,1]$; and $\left\|A g_{m}-C_{m}\right\| \leqq \frac{1}{m}$. Thus $\left\|f-C_{m}\right\|<\frac{2}{m}$, for each $m$ and $f$ is almost constant. It follows that $\mathfrak{A}^{\prime}$ is the scalars and the given representation, which we refer to as the Lebesgue measure representation of $\mathfrak{A}$, is irreducible.

We note next that $\alpha$ is weakly-inner in this representation. Let $f_{j}^{(n)}$ be the characteristic function of $\left[\frac{j-1}{2^{n}}, \frac{j}{2^{n}}\right]$. Then

$$
U_{m}\left(f_{j_{0}}^{(n)}\right)=\left(\sum_{j+k=2^{m}+1} E_{j k}^{(m)}\right)\left(f_{j_{0}}^{(n)}\right)=U_{n}\left(f_{j_{0}}^{(n)}\right)
$$


for all $m \geqq n$. Thus $\left(U_{m}\right)$ converges on all finite linear combinations of the $f_{j}^{(n)}$. Since such combinations lie dense in $\mathscr{L}_{2}(0,1)$ and $\left\|U_{m}\right\|=1$ for all $m$, the $U_{m}$ converge strongly to some operator $U$ on $\mathscr{H}$. Now $U_{m}^{2}=I$ and multiplication is jointly continuous on bounded sets of operators in the strong-operator topology. Thus $U^{2}=I$ and since each $U_{m}$ is isometric $U$ is. It follows that $U$ is a unitary operator. Again, since $U_{m} E_{j k}^{(n)} U_{m}$ $=U_{n} E_{j k}^{(n)} U_{n}=\alpha\left(E_{j k}^{(n)}\right)$ for all $m \geqq n$ and all $j, k, n, U E_{j k}^{(n)} U=\alpha\left(E_{j k}^{(n)}\right)$ for all $j, k, n$. Thus $\alpha$ is induced by the unitary operator $U$ in the Lebesgue measure representation of $\mathfrak{A}$, and, since this is an irreducible representation, $\alpha$ is weakly inner. It can be verified readily that $U$ is the unitary operator defined by $(U f)(t)=f(1-t)$ for each $f$ in $\mathscr{L}_{2}(0,1)$.

For our representation in which $\alpha$ is not extendable, we choose as our Hilbert space $\mathscr{H}_{0}$ the space $\mathscr{L}_{2}([0,1), \mu)$ where the measure $\mu$ on $[0,1)$ is defined by assigning to each Borel subset the number of dyadic rational points it contains. (In this way we make each dyadic rational point in $[0,1)$ an atom for $\mu$ with measure 1.) The matrix units $E_{j k}^{(n)}$ are defined in precisely the same way as in the Lebesgue measure representation except that the half-open intervals $\left[\frac{j-1}{2^{n}}, \frac{j}{2^{n}}\right)$ are used in place of the closed intervals $\left[\frac{j-1}{2^{n}}, \frac{j}{2^{n}}\right]$. The functions 1 at a dyadic rational in $[0,1)$ and 0 off it form an orthonormal basis for $\mathscr{H}_{0}$, and the one-dimensional projections with these in their range are intersections of diagonal matrix units of $\mathfrak{A}$ in the given representation. Moreover, the partial isometries between these one-dimensional projections induced by mapping one dyadic rational onto another are the weak-operator limits of the matrix units mapping the ranges of these diagonal matrix units on to one another. It follows that $\mathfrak{A}$ has weak-operator closure all bounded operators in this representation and that this representation is, accordingly, irreducible. Now $\alpha\left(E_{11}^{(n)}\right)=E_{2^{n} 2^{n}}^{(n)}$ for each $n$; and while $\bigcap_{n} E_{11}^{(n)}$ is the onedimensional projection with the function 1 at 0 and 0 elsewhere in its range, $\bigcap_{n} E_{2^{n} 2^{n}}^{(n)}=0$ in this representation. Thus $\alpha$ is not extendable.

In the example which follows, we illustrate the fact that there are automorphisms of $C^{*}$-algebras which are $\pi$-inner without being actual inner automorphisms of the algebra.

Example b. Let $\mathfrak{A}$ be the $C^{*}$-algebra of compact operators on separable Hilbert space with the identity $I$ adjoined, so that each operator in $\mathfrak{A}$ has the form $a I+C$ with $a$ some scalar and $C$ a compact operator. The abstract $C^{*}$-algebra associated with $\mathfrak{A}$ has just two irreducible representations - the given one through which we have defined $\mathfrak{A}$ and the one-dimensional representation, $a I+C \rightarrow a$. Any other representation $\varphi$ of $\mathfrak{A}$ is a direct sum of copies of these two representations, for if $\varphi$ is not faithful $\varphi(\mathfrak{Q})$ is the scalars. Thus, for general $\varphi$, there is a maximal 
projection $E^{\prime}$ in $\varphi(\mathfrak{U})^{\prime}$ such that $\varphi(\mathfrak{U}) E^{\prime}=\left\{\lambda E^{\prime}\right\}$; and each non-zero subprojection $F^{\prime}$ of $I-E^{\prime}$ is such that $A \rightarrow \varphi(A) F^{\prime}$ is a faithful representation of $\mathfrak{A}$. Combining this with the fact that each faithful representation $\psi$ of $\mathfrak{A}$ has the faithful irreducible representation as a subrepresentation and using Zorn's lemma establishes the assertion about $\varphi$. For this, note that $\psi(E)$ is minimal in $\psi(\mathfrak{A})^{-}$since $\psi(E) \psi(A) \psi(E)=a E$ with $a$ a scalar for all $A$ in $\mathfrak{A}$ if $E$ is a one-dimensional projection in $\mathfrak{A}$, so that $\psi(\mathfrak{A})^{\prime}$ contains minimal projections. Choosing a maximal orthogonal family of such projections, if $\psi(\mathfrak{A})$ restricts to scalars on each then the restriction of $\psi(\mathfrak{A})$ to the complement of their union is faithful since $\psi$ is; so that this complement contains a minimal projection - contradicting maximality of the family chosen. Thus $\psi$ has the faithful irreducible representation of $\mathfrak{A}$ as a subrepresentation.

It follows that $\psi(\mathfrak{U})$ - has a central projection $Q$ such that $\psi(\mathfrak{A})-(I-Q)$ $=\{\lambda(I-Q)\}$ and $\psi(\mathfrak{U})-Q$ acting on $Q\left(\mathscr{H}_{1}\right)$ is a factor of type $I_{\infty}$. Since a state of $\psi(\mathfrak{A}) Q$ is normal if and only if it does not annihilate all the compact operators, each automorphism $\alpha$ of $\psi(\mathfrak{A})$ transforms normal states onto normal states and extends to an automorphism $\bar{\alpha}$ of $\psi(\mathfrak{A})^{-}$. Of course, $\bar{\alpha}$ maps $\psi(\mathfrak{A})-Q$, a factor of type $I_{\infty}$, onto itself and $\psi(\mathfrak{I})-(I-Q)$, scalars, onto itself, so that $\bar{\alpha}$ is inner. Now each unitary $U$ on $\mathscr{H}$ induces an automorphism of $\mathfrak{A}$; and since $\mathfrak{A}$ acts irreducibly on $\mathscr{H}$, this automorphism is not inner unless $U$ is in $\mathfrak{A}$. Thus $\mathfrak{A}$ admits non inner, permanently weakly-inner automorphisms.

In the example just discussed, with $\mathfrak{A}$ in its faithful irreducible representation, each automorphism is induced by a unitary operator $U$ and each unitary operator induces such an automorphism of $\mathfrak{A}$. With $U=\exp i H$, the automorphism induced by $U$ lies on the one-parameter group of automorphisms of $\mathfrak{A}$ induced by the unitary operators expit $H$, $t$ real. Thus $\gamma(\mathfrak{U})=\alpha(\mathfrak{U})$, in this case. In the example to follow we discuss a $C^{*}$-algebra which is not GCR (not "post-liminaire" c.f. [7; $\S 4.2,4.3$, pp. $86-87]$ ) and use the results of Theorem 7 to produce $\pi$-inner automorphisms that are not inner in a situation where there are automorphisms that are not $\pi$-inner yet weakly-inner in some faithful representation.

Example c. Let $\mathscr{M}$ be a factor of type $I I_{1}$ acting on a (separable) Hilbert space $\mathscr{H}$ and having coupling 1 (e.g. the von Neumann algebra generated by the left regular representation of the free group on two generators). Let $\mathscr{C}$ be the algebra (ideal in $\mathscr{B}(\mathscr{H})$ ) of compact operators. Then the set $\{A+C: A$ in $\mathscr{M}, C$ in $\mathscr{C}\}$ is a self-adjoint operator algebra. Moreover it is a $C^{*}$-algebra $\mathfrak{A}$ since it is norm closed. We see this by noting that the "angle" between the closed linear subspaces $\mathscr{C}$ and $\mathscr{M}$ of $\mathscr{B}(\mathscr{H})$ is greater than 0 ; for if $A$ in $\mathscr{M}$ has norm 1 , one of $\left(A+A^{*}\right) / 2$ and $\left(A-A^{*}\right) / 2 i$ has norm at least $1 / 2$. Let $\varrho_{0}$ be a pure state of $\mathscr{M}$ 
assigning $a$ with $|a| \geqq \frac{1}{2}$ to one of these operators and let $\varrho$ be a pure state extension of $\varrho_{0}$ to $\mathscr{B}(\mathscr{H})$. Since no pure state of $\mathscr{M}$ (a $I I_{1}$ factor) is a vector state, $\varrho$ is not a vector state; and, therefore, annihilates $\mathscr{C}$. Thus, for each $C$ in $\mathscr{C}, \frac{1}{2} \leqq|\varrho(A-C)|=|\varrho(A)| \leqq\|A-C\|$; and $\mathscr{M}+\mathscr{C}$ is closed.

Since $\mathscr{M}$, as represented, has coupling 1 , each automorphism $\alpha$ is implemented by a unitary operator $U$; and $\alpha_{0}(A+C)$ defined as $U^{*}(A+C) U$ makes $\alpha_{0}$ an automorphism of $\mathfrak{A}$ (of course, weakly inner, since $\left.\mathfrak{A}^{-}=\mathscr{B}(\mathscr{H})\right)$. Now $\mathscr{A} / \mathscr{C}$ is $\mathscr{M}$; so that the given representation of $\mathscr{M}$ combined with this quotient mapping, provides a representation $\varphi$ of $\mathfrak{A}$ on $\mathscr{H}$. The faithful representation $\iota \oplus \varphi$ of $\mathfrak{A}$ on $\mathscr{H} \oplus \mathscr{H}$ carries $\alpha_{0}$ on $\mathfrak{A}$ onto the automorphism $\beta$ defined by

$$
\beta(\{A+C, A\})=\left\{\alpha_{0}(A)+\alpha_{0}(C), \alpha_{0}(A)\right\},
$$

for each $A$ in $\mathscr{M}$ and $C$ in $\mathscr{C}$. Since $\iota$ and $\varphi$ are disjoint $(\iota$ being irreducible has no proper subrepresentations and $\varphi$ being a $I I_{1}$ factor representation has no irreducible - indeed, no type $I$ - subrepresentations $),(\iota \oplus \varphi)(\mathfrak{A})^{-}$ $=\mathscr{B}(\mathscr{H}) \oplus \mathscr{M}$. If $\beta$ (that is, $\alpha_{0}$ in the representation $\iota \oplus \varphi$ ) were weakly inner the unitary operator implementing it would have a (unitary) component in $\mathscr{M}$ which implements $\alpha$. For an example of an automorphism $\alpha_{0}$ of $\mathfrak{A}$ which, while weakly-inner in the given representation of $\mathfrak{A}$, is not weakly-inner in that given by $\iota \oplus \varphi$, we have only to choose for $\alpha$ one of the (many) outer automorphisms of $\mathscr{M}$ (compare [6; Exercise 15, p. 308]).

To construct $\pi$-inner automorphisms of $\mathfrak{A}$ which are not inner, let $U$ be a unitary operator on $\mathscr{H}$ in $\mathscr{M}^{\prime}$ with $\|U-I\|<1$ and $U$ not a scalar. Then $U$ induces an automorphism $\alpha$ of $\mathfrak{A}$ such that $\|\alpha-\iota\|<2$; so that $\alpha$ is $\pi$-inner (see Theorem 7 ). However $\alpha$ is not inner since $\mathfrak{A}$ acts irreducibly and $U$ is not in $\mathfrak{A}$. For suppose $U=A+C$ is in $\mathfrak{A}$, with $A$ in $\mathscr{M}$ and $C$ in $\mathscr{C}$. For each $A^{\prime}$ in $\mathscr{M}^{\prime}, C A^{\prime}-A^{\prime} C=U A^{\prime}-A^{\prime} U$, so $C A^{\prime}-A^{\prime} C$ is a compact operator in $\mathscr{M}^{\prime}$ and is therefore zero. Hence, $U \in \mathscr{M} \cap \mathscr{M}^{\prime}$ and $U$ is a scalar, contrary to our choice of $U$.

With some slight additional effort we can analyze a faithful representation $\varphi$ of $\mathfrak{A}$ sufficiently to establish that each automorphism $\alpha$ of $\mathfrak{A}$ which is the identity on $\mathscr{M}$ is $\pi$-inner. In fact, as in Example $b, \varphi(\mathfrak{A})^{-}$ has minimal projections, so that $\varphi(\mathfrak{U})^{\prime}$ also has minimal projections restrictions to which produce the faithful irreducible representation of $\mathfrak{A}$ (cf. [7; Corollary 4.1.10, p. 85]). Such a minimal projection $E^{\prime}$ has central carrier $Q$, a minimal central projection. Now, restriction of $\varphi(\mathfrak{A})$ to $I-Q$ cannot be a faithful representation of $\mathfrak{U}$ for then, as just noted, it would have a faithful irreducible subrepresentation inequivalent to $\varphi(\mathfrak{U}) E^{\prime}$, since they are separated by the orthogonal central 
projections $Q$ and $I-Q$, contradicting the uniqueness of the faithful irreducible representation of $\mathfrak{A}$. Thus $\varphi(\mathscr{C})(I-Q)=(0)$, since $\mathscr{C}$ is the unique proper closed two sided ideal in $\mathfrak{A}$. Hence $\varphi \propto \varphi^{-1}$ is the identity on $\varphi(\mathfrak{A})(I-Q)$ after composition with restriction to $I-Q$; and is induced by the unitary operator (in $\varphi(\mathfrak{A})^{-}$) which acts as the identity on $(I-Q) \mathscr{H}_{0}$ and which induces the restriction of $\varphi \alpha \varphi^{-1}$ to $\varphi(\mathfrak{A}) Q$ on $Q \mathscr{H}_{0}$, where $\mathscr{H}_{0}$ is the representation space of $\varphi$.

By use of Lemma 2 one can give a shorter proof of the same result. For any automorphism $\alpha$ of $\mathfrak{A}$ which is the identity on $\mathscr{M}$ is implemented by a unitary operator $U=\exp i H$, with $H=H^{*}$ in $\mathscr{M}^{\prime}$. With $\alpha_{t}$ the automorphism induced on $\mathfrak{A}$ by $\exp i t H, t \rightarrow \alpha_{t}$ is a norm-continuous one-parameter group in $\alpha(\mathfrak{A})$ which contains $\alpha$, whence $\alpha$ is $\pi$-inner (Lemma 2).

In the class of examples which follow, we exhibit instances in which all possible equalities and inequalities consistent with the inclusion $\gamma(\mathfrak{U}) \leqq \iota_{0}(\mathfrak{U}) \leqq \pi(\mathfrak{U})$ occur among the groups $\gamma(\mathfrak{U}), \iota_{0}(\mathfrak{U}), \pi(\mathfrak{U})$ - the first inclusion being a special feature of this class of examples (c.f. Example $b$ and the remarks following).

Example d. Throughout this discussion $\mathscr{A}$ is an abelian $C^{*}$-algebra isomorphic to $C(X)$ with $X$ a compact-Hausdorff space (the pure state space of $\mathscr{A}) ; \mathscr{M}_{n}$ is the algebra of operators $(n \times n$ complex matrices) acting on $n$-dimensional complex Hilbert space and $\mathfrak{A}$ is the $C^{*}$-algebra $\mathscr{A} \otimes \mathscr{M}_{n}$. There are two convenient ways of viewing $\mathfrak{A}$, as $n \times n$ matrices with entries in $\mathscr{A}$ (or $C(X)$ ) and as continuous functions on $X$ with values in $\mathscr{M}_{n}$. The center $\mathscr{C}$ of $\mathfrak{A}$ is the set of matrices whose only nonzero entries consist of a single $A$ in $\mathscr{A}$ at each diagonal position (equivently, the continuous mappings of $X$ into scalars in $\mathscr{M}_{n}$ ). We denote by $\alpha_{c}(\mathfrak{U})$ those automorphisms of $\mathfrak{U}$ which leave each element of $\mathscr{C}$ fixed.

We prove first that $\alpha_{c}(\mathfrak{U})$ and $\pi(\mathfrak{U})$ coincide. Since in each faithful representation of $\mathfrak{A}$ an element of $\pi(\mathfrak{U})$ leaves the center of the weak operator closure of $\mathfrak{A}$ and a fortiori $\mathscr{C}$ elementwise fixed, we have $\pi(\mathfrak{U}) \leqq \alpha_{c}(\mathfrak{U})$. Suppose that $\alpha$ is in $\alpha_{c}(\mathfrak{A})$. With $E_{j k}, j, k=1, \ldots, n$ matrix units of $\mathscr{M}_{n}$ and $\alpha\left(I \otimes E_{j k}\right)=B_{j k} ;$ we have $\alpha\left(\sum_{j, k} A_{j k} \otimes E_{j k}\right)$ $=\Sigma_{j, k}\left(A_{j k} \otimes I\right) B_{j k}$, since $\alpha(A \otimes I)=A \otimes I$. Since multiplication by $B_{j k}$ and $A_{j k} \rightarrow A_{j k} \otimes I$ are strong-operator continuous; $\alpha$ (and, similarly, $\left.\alpha^{-1}\right)$ is strong-operator continuous, has an extension $\bar{\alpha}$ to the weakoperator closure $\mathscr{A}^{-} \otimes \mathscr{M}_{n}$ of $\mathfrak{A}$, and $\bar{\alpha}$ is an automorphism of $\mathfrak{A}-$. For this we note that the faithful representation of $\mathfrak{A}$ under consideration is unitarily equivalent to $\mathscr{A} \otimes \mathscr{M}_{n}$ acting on the $n$-fold direct sum of a Hilbert space on which $\mathscr{A}$ is represented faithfully. Now the commutant of $\mathfrak{A}^{-}$is $\mathscr{A}^{\prime} \otimes I$, and $\bar{\alpha}(A \otimes I)=A \otimes I$ for $A$ in $\mathscr{A}^{-}$since this is true for $A$ in the strong operator dense subset $\mathscr{A}$ of $\mathscr{A}^{-}$and $\bar{\alpha}$ is strong-operator continuous. Thus $\bar{\alpha}$ leaves the center of $\mathfrak{A}^{-}$elementwise fixed, and $\bar{\alpha}$ is 
inner since $\mathfrak{A}^{-}$is of type $I$. It follows that $\alpha$ is in $\pi(\mathfrak{A})$. Hence $\pi(\mathfrak{Z})$ $\geqq \alpha_{c}(\mathfrak{Q})$, so that $\pi(\mathfrak{R})=\alpha_{c}(\mathfrak{Q})$.

With $\alpha$ in $\pi(\mathfrak{A})$ and $\varrho$ a point of $X$ (i.e. a pure state of $\mathscr{A}$ ) a homomorphism $\varphi_{\varrho}$ of $\mathscr{A} \otimes \mathscr{M}_{n}$ onto $\mathscr{M}_{n}$ is determined by $\varphi_{\varrho}(A \otimes B)=\varrho(A) B$. With $B$ in $\mathscr{M}_{n}$ and $\alpha(\varrho)(B)$ defined as $\varphi_{\varrho}(\alpha(I \otimes B)), \alpha(\varrho)$ is an isomorphism of $\mathscr{M}_{n}$ into $\mathscr{M}_{n}$; since $\varphi_{\varrho}(\alpha(I \otimes I))=I$ and the closed 2 -sided ideal generated by $I \otimes B$ is $\mathfrak{A}$, if $B \neq 0$. From the finite dimensionality of $\mathscr{M}_{n}$, we conclude that $\alpha(\varrho)$ is an automorphism of $\mathscr{M}_{n}$ and that all topological linear structures on the (bounded) linear operators over $\mathscr{M}_{n}$ are equivalent. Thus, in order to establish the norm continuity of $\varrho \rightarrow \alpha(\varrho)$ it suffices to establish the continuity of $\varrho \rightarrow \alpha(\varrho)(B)$ for each fixed $B$ in $\mathscr{M}_{n}$. If $\alpha(I \otimes B)=\sum_{j, k=1}^{n} A_{j k} \otimes E_{j k}$ with $A_{j k}$ in $\mathscr{A}$, then $\alpha(\varrho)(B)=\sum_{j, k=1}^{n} \varrho\left(A_{j k}\right) E_{j k}$; and the continuity in question follows from the definition of the $w^{*}$-topology on $X$.

Conversely, if $\varrho \rightarrow \alpha(\varrho)$ is an arbitrary continuous mapping of $X$ into $\alpha\left(\mathscr{M}_{n}\right)$, with $B$ in $\mathscr{M}_{n}$ and $\alpha(\varrho)(B)=\sum_{j, k=1}^{n} \hat{A}_{j k}(\varrho) E_{j k}$, we have $\varrho \rightarrow E_{j j} \alpha(\varrho)(B) E_{k k}=\hat{A}_{j k}(\varrho) E_{j k}$ is continuous; so that each $\hat{A}_{j k}$ is a continuous complex-valued function on $X$ and corresponds to a (unique) operator $A_{j k}$ in $\mathscr{A}$. Defining $\alpha(A \otimes B)$ to be $\sum_{j, k=1}^{n} A A_{j k} \otimes E_{j k}$ determines an automorphism $\alpha$ of $\mathscr{A}$ in $\pi(\mathfrak{R})\left(=\alpha_{c}(\mathfrak{R})\right)$. The identity $(\alpha \beta)(\varrho)$ $=\alpha(\varrho) \beta(\varrho)$ is valid, justifying the notation ' $\alpha(\varrho)$ ' and proving that the correspondence between elements of $\pi(\mathfrak{H})$ and continuous mappings of $X$ into $\alpha\left(\mathscr{M}_{n}\right)$ is a group isomorphism when this second set is provided with pointwise multiplication through the group structure of $\alpha\left(\mathscr{M}_{n}\right)$. Henceforth we pass from the elements of $\pi(\mathfrak{A})$ to the continuous mappings of $X$ into $\alpha\left(\mathscr{M}_{n}\right)$ without comment.

Since each automorphism of $\mathscr{M}_{n}$ is inner and the only unitary operators in $\mathscr{M}_{n}$ inducing the identity automorphism of $\mathscr{M}_{n}$ are the scalars of modulus $1, \alpha\left(\mathscr{M}_{n}\right) \approx U(n) / T_{1}$, where $U(n)$ is the group of unitary operators in $\mathscr{M}_{n}$ and $T_{1}$, its center, is the circle group. Let $p$ be the natural mapping of $U(n)$ onto $U(n) / T_{1}$. If $\alpha$ in $\pi(\mathfrak{A})$ is inner there is a unitary operator $U$ in $\mathfrak{A}$ which implements it. Let $\tilde{\alpha}(\varrho)$ be $\varphi_{\varrho}(U)$, an element of $U(n)$. Again $\varrho \rightarrow \tilde{\alpha}(\varrho)$ is a continuous mapping of $X$ into $U(n)$ and $p \tilde{\alpha}=\alpha$. Conversely, if $\tilde{\alpha}$ is a continuous mapping of $X$ into $U(n)$, $\alpha=p \tilde{\alpha}$ is a continuous mapping of $X$ into $U(n) / T_{1}$, i.e. an element of $\pi(\mathfrak{A})$, while $\tilde{\alpha}$ is an element $U$ of the unitary group of $\mathscr{A}$ which implements $\alpha$. Thus $\iota_{0}(\mathfrak{Q})$ is the group of continuous mappings of $X$ into the base space $U(n) / T_{1}$ which can be "lifted" to the bundle $U(n)$ (with projection 
$p$, fibre and group $T_{1}$ ). From Theorem 7, each element $\gamma$ of the connected component of the identity $\gamma(\mathfrak{A})$ of $\alpha(\mathfrak{A})$ is a product $\gamma_{1} \ldots \gamma_{m}$ where $\gamma_{j}=\gamma_{j}(1)$ and $t \rightarrow \gamma_{j}(t)$ is a norm continuous one-parameter group in $\alpha(\mathfrak{A})$. Thus, with $\Gamma(\varrho, t)=\left(\gamma_{1}(t)\right)(\varrho) \ldots\left(\gamma_{m}(t)\right)(\varrho), \Gamma$ is a homotopy of $\gamma$ and $\varrho \rightarrow \gamma(\varrho, 0)=T_{1} \ldots T_{1}=T_{1}$, i.e. of $\gamma$ and the constant mapping of $X$ onto the identity element of $U(n) / T_{1}$. Of course this constant mapping lifts to $U(n)$; and the Covering Homotopy Theorem [29; Theorem 11.7, p. 54] tells us that the homotopy under consideration can be covered by a homotopy of this lifted constant mapping in the bundle space $U(n)$. This homotopy in the bundle provides a lifting of $\gamma$ from $U(n) / T_{1}$ to $U(n)$. Thus $\gamma$ is in $\iota_{0}(\mathfrak{R})$ and $\gamma(\mathfrak{A}) \leqq \iota_{0}(\mathfrak{A}) \leqq \pi(\mathfrak{A})$ $=\alpha_{c}(\mathfrak{Q})$.

From this same argument, if $\alpha$ and $\beta$ in $\pi(\mathfrak{A})$ are in the same coset of $\gamma(\mathfrak{A})$, say $\alpha=\beta \gamma$ with $\gamma$ in $\gamma(\mathfrak{A})$, then a homotopy of $\gamma$ with the constant mapping of $X$ onto $T_{1}$ in $U(n) / T_{1}$ provides a homotopy between $\alpha$ and $\beta$. Conversely, if $\alpha$ and $\beta$ are homotopic and $F: X \times[0,1] \rightarrow U(n) / T_{1}$, $F(\varrho, 0)=\alpha(\varrho), F(\varrho, 1)=\beta(\varrho)$ is a homotopy, then $\beta^{-1} F$ defined by $\left(\beta^{-1} F\right)(\varrho, t)=\beta^{-1}(\varrho) F(\varrho, t)$ (group product in $\left.U(n) / T_{1}\right)$ is a homotopy of $\beta^{-1} \alpha$ with the constant mapping (onto $T_{1}$ ). Hence $\beta^{-1} \alpha$ lies in $\gamma(\mathfrak{Q})$ (it is connected to the identity automorphism by the "arc" which is the homotopy just described). Thus $\pi(\mathfrak{Q}) / \gamma(\mathfrak{A})$ is the group of homotopy classes of mappings of $X$ into $U(n) / T_{1}$, the product of two such classes being formed by multiplying any two representatives pointwise using the multiplication in $U(n) / T_{1}$ and passing to the class of the result. Since $\gamma(\mathfrak{Z}) \leqq \iota_{0}(\mathfrak{A})$, each $\gamma(\mathfrak{A})$-coset of an element $\alpha$ of $\iota_{0}(\mathfrak{U})$ consists of elements in $\iota_{0}(\mathfrak{Q})$. From the foregoing, this coset is the class of mappings of $X$ into $U(n) / T_{1}$ homotopic to $\alpha$. Thus each $\beta$ homotopic to $\alpha$ lies in $\iota_{0}(\mathfrak{A})$ (can be lifted to $U(n)$ - the Covering Homotopy argument gives this same result directly), and $\iota_{0}(\mathfrak{R}) / \gamma(\mathfrak{Q})$ is the group of homotopy classes of continuous mappings of $X$ into $U(n) / T_{1}$ which can be lifted to $U(n)$.

Applying these general topological identifications of $\gamma(\mathfrak{A}), \iota_{0}(\mathfrak{A})$, $\pi(\mathfrak{Z})$ and their quotients to specific choices of $X$, we note first that if $X$ is contractible (to a point) - for example, if $X$ is the unit ball in $n$-space then each continuous mapping of $X$ is homotopic to a constant mapping, $\pi(\mathfrak{Q}) / \gamma(\mathfrak{Q})$ has a single element, so that $\gamma(\mathfrak{R}), \iota_{0}(\mathfrak{H})$ and $\pi(\mathfrak{Q})$ coincide in this case. Specifically, if $\mathscr{A}$ is $C([0,1])$ and $\mathfrak{A}$ is $\mathscr{A} \otimes \mathscr{M}_{n}, \gamma(\mathfrak{A})$ $=\iota_{0}(\mathfrak{R})=\pi(\mathfrak{R})$.

At the other extreme, we show that if $\mathscr{A}$ is $C\left(U(n) / T_{1}\right)$ and $\mathfrak{A}$ is $\mathscr{A} \otimes \mathscr{M}_{n}$, then $\gamma(\mathfrak{U}) \varsubsetneqq \iota_{0}(\mathfrak{A}) \varsubsetneqq \pi(\mathfrak{U})$. The last inequality is established by noting that the identity mapping of $U(n) / T_{1}$ onto $U(n) / T_{1}$ cannot be lifted to $U(n)$; in other words, the bundle $\left\{U(n), p, U(n) / T_{1}, T_{1}, T_{1}\right\}$ does not have a cross section. To see this note that $U(n)$ is homeo- 
morphic to $T_{1} \times S U(n)$ and has fundamental group $\pi_{1}(U(n))$ isomorphic to $\mathbb{Z}$, the additive group of integers [4; Proposition 7, p. 61], where $S U(n)$ the special unitary group is the group of unitary operators in $\mathscr{M}_{n}$ having determinant 1 . Since $U(n)$ is $T_{1} \cup S U(n)$ and $T_{1} \cap S U(n)$ is $\mathbb{Z}_{n}$ the group of multiples of $I$ by $n^{t h}$ roots of unity, the second isomorphism theorem of group theory tells us that $U(n) / T_{1}$ is isomorphic (as a topological group) to $S U(n) / \mathbb{Z}_{n}$ (here, $\mathbb{Z}_{n}$ is the center of $S U(n)$ ). Now $S U(n)$ is simply connected and $\mathbb{Z}_{n}$ is a discrete subgroup in (equal to) the center of $S U(n)$. Thus $0 \rightarrow \mathbb{Z}_{n} \rightarrow S U(n) \rightarrow S U(n) / \mathbb{Z}_{n} \rightarrow 0$ is a covering mapping and $\pi_{1}\left(S U(n) / \mathbb{Z}_{n}\right)\left(=\pi_{1}\left(U(n) / T_{1}\right)\right) \approx \mathbb{Z}_{n}$ (cf. [4; Proposition 7, p. 54 and Proposition 6, p. 60]). If our bundle admits a cross section then $\pi_{1}(U(n))(\approx \mathbb{Z})$ has a subgroup isomorphic to $\pi_{1}\left(U(n) / T_{1}\right)\left(\approx \mathbb{Z}_{n}\right)$ (cf. [29; 17.7, p. 92], actually $\pi_{1}(U(n))$ would be the direct sum of $\pi_{1}\left(U(n) / T_{1}\right)$ and $\pi_{1}\left(T_{1}\right)$ since it is abelian). Of course this is not the case since $\mathbb{Z}$ has no torsion. Thus the identity mapping of $U(n) / T_{1}$ onto $U(n) / T_{1}$ does not lift and provides an element $\alpha$ in $\pi(\mathfrak{A})$ not in $\iota_{0}(\mathfrak{A})$.

We exhibit, next, an essential mapping $\alpha$ of $U(n) / T_{1}$ into $U(n) / T_{1}$ which lifts to a mapping of $U(n) / T_{1}$ into $U(n)$. Thus $\alpha$ is an element of $i_{0}(\mathfrak{A})$ not in $\gamma(\mathfrak{U})$. To describe $\alpha$ we use the representation of $U(n) / T_{1}$ as $S U(n) / \mathbb{Z}_{n}$ and of $U(n)$ as the product $T_{1} \times S U(n)$ noted above. From the form of the representation $T_{1} \times S U(n), i: U \rightarrow(1, U)$ is just the inclusion mapping of $S U(n)$ into $U(n)$. Let $q$ be the natural mapping of $S U(n)$ onto $S U(n) / \mathbb{Z}_{n}$ and $s: U \rightarrow U^{n}$ a mapping of $S U(n)$ into $S U(n)$. Since $q$ is open and $s$ is continuous and maps $\mathbb{Z}_{n}$ onto $I$, the mapping $r: U \mathbb{Z}_{n} \rightarrow U^{n}$ of $S U(n) / \mathbb{Z}_{n}$ into $S U(n)$ is well-defined, satisfies $r q=s$ and is continuous. With $t=i r$ mapping $S U(n) / \mathbb{Z}_{n}$ into $U(n), t$ is continuous and the diagram

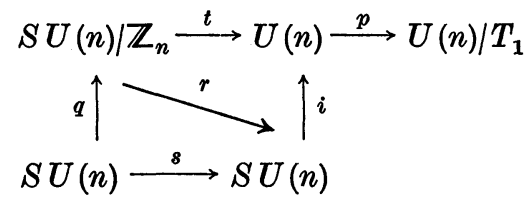

is commutative. We assert that $\alpha(=p t)$ is an essential mapping (i.e. not homotopic to a constant mapping) of $U(n) / T_{1}$ into $U(n) / T_{1}$. Suppose the contrary. With $f$ a continuous mapping of $X$ into $Y$, we denote by $f_{*}$ the induced homomorphism of $\pi_{m}(X)$ into $\pi_{m}(Y)$ (cf. $[29 ; 15.5$, p. 75]). Since $p i s=p t q$ and $p t$ is inessential $p_{*} t_{*}\left(=(p t)_{*}\right)$ is 0 on $\pi_{m}\left(S U(n) / \mathbb{Z}_{n}\right)$; so that $p_{*} i_{*} s_{*}\left(=p_{*} t_{*} q_{*}\right)$ is 0 on $\pi_{m}(S U(n))$ (cf. $\left[29 ; 15.6,2^{\circ}\right.$ and $5^{\circ}$, p.76]). Let $f$ be a mapping of $S^{3}$, the 3-sphere, into $S U(n)$. Then $s f\left(=f^{n}\right)$ is homotopic to $n f$ (in the sense of homotopy addition) [29; 16.7, p. 88]. From [29; 17.8, p. 93, 25.1, p. 131 and 25.4, p. 132], 
$\mathbb{Z} \approx \pi_{3}(U(n)) \approx \pi_{3}\left(T_{1}\right)+\pi_{3}(S U(n)) \approx \pi_{3}(S U(n))$. With $f$ a representative of a generator $z$ of $\pi_{3}(S U(n)), n f$ and, hence, $s f$ are representatives of $n z\left(\neq 0\right.$ since $\left.\pi_{3}(S U(n)) \approx \mathbb{Z}\right)$. But $s f$ is a representative of $s_{*}(z)$; so that $s_{*}(z)=n z \neq 0$. On the other hand, $i_{*}$ is injective since it is induced by a (trivial) bundle cross section $[29 ; 17.7$, p. 92 , see the proof], and $p_{*}$ is injective on $\pi_{3}(U(n))$ from the exactness of the homotopy sequence of the bundle $\left\{U(n), p, U(n) / T_{1}, T_{1}, T_{1}\right\}$ as applied to the portion $\cdots \rightarrow \pi_{3}\left(T_{1}\right) \rightarrow \pi_{3}(U(n)) \stackrel{p_{*}}{\longrightarrow} \pi_{3}\left(U(n) / T_{1}\right) \stackrel{\Delta}{\longrightarrow} \pi_{2}\left(T_{1}\right) \rightarrow \cdots$ (noting that $\pi_{3}\left(T_{1}\right)=\pi_{2}\left(T_{1}\right)=0$ together with exactness shows that $p_{*}$ is an isomorphism of $\pi_{3}(U(n))$ onto $\left.\pi_{3}\left(U(n) / T_{1}\right)\right)$ [29; 17.3 and 17.4, p. 91]. Since $i_{*} s_{*}(z)$ is a non-zero element of $\pi_{3}(U(n)), p_{*} i_{*} s_{*}(z) \neq 0$ contradicting the earlier conclusion that $p_{*} i_{*} s_{*}$ is 0 on each $\pi_{m}(S U(n))$. Thus $\alpha(=p t)$ is essential and provides an element of $\iota_{0}(\mathfrak{A})$ (since it can be lifted to $t$ ) not in $\gamma(\mathfrak{A})$.

For our next illustration, we take $X$ to be $T_{1}$ and $\alpha$ a continuous mapping of $T_{1}$ into $U(n) / T_{1}\left(\approx S U(n) / \mathbb{Z}_{n}\right)$ which represents a non-zero element in $\pi_{1}\left(U(n) / T_{1}\right)\left(\approx \mathbb{Z}_{n}\right)$. Then $\alpha$ is not in $\gamma(\mathfrak{U})$. However each continuous mapping $\beta$ of $T_{1}$ into $U(n) / T_{1}$ can be lifted to $U(n)$. To see this choose a fixed triangulation of $U(n) / T_{1}$ as a complex $K$. Since the fibre $T_{1}$ is arcwise connected, the bundle over the 1-skeleton $K^{1}$ of $K$ has a cross section (cf. [29; last statement, p. 148]), so that each simplicial mapping of a space $X$ into $K^{1}$ can be lifted. In particular each simplicial mapping of $T_{1}$ into $K$ can be lifted. Now $\beta$ is homotopic to a simplicial mapping of $T_{1}$ into $K$ (from the Simplicial Approximation Theorem) so that the Covering Homotopy Theorem [29; 11.7 p. 54] guarantees a lifting of $\beta$. We conclude that, with $\mathscr{A}$ taken as $C\left(T_{1}\right)$ and $\mathfrak{A}$ as $\mathscr{A} \otimes \mathscr{M}_{n}$, $\gamma(\mathfrak{U}) \nsubseteq \iota_{0}(\mathfrak{U})=\pi(\mathfrak{U})$. In this example $\iota_{0}(\mathfrak{U}) / \gamma(\mathfrak{U})(=\pi(\mathfrak{U}) / \gamma(\mathfrak{U}))$, the homotopy classes of mappings of $X$ into $U(n) / T_{1}\left(=S U(n) / \mathbb{Z}_{n}\right)$, is just $\pi_{1}\left(S U(n) / \mathbb{Z}_{n}\right)$ which we have identified as isomorphic to $\mathbb{Z}_{n}$.

To illustrate the possibility that $\gamma(\mathfrak{U})=\iota_{0}(\mathfrak{A})$ with $\iota_{0}(\mathfrak{U})$ a proper subgroup of $\pi(\mathfrak{A})$, we exhibit a compact space $X$ and a (continuous) mapping of $X$ into $U(2) / T_{1}$ which cannot be lifted to $U(2)$, so that this mapping is an element of $\pi(\mathfrak{A})$ not in $\iota_{0}(\mathfrak{U})$; while each mapping of $X$ into $U(2)$ is inessential, hence (by projecting the homotopy) each mapping of $X$ into $U(2) / T_{1}$ which can be lifted to $U(2)$ is inessential from which, $\gamma(\mathfrak{A})=\iota_{0}(\mathfrak{U})$. Recall that $U(2) / T_{1} \approx S U(2) / \mathbb{Z}_{2}$ and that each element $U$ of $S U(2)$ has the form $\left(\begin{array}{lr}a & -\bar{b} \\ b & \bar{a}\end{array}\right)$, where $|a|^{2}+|b|^{2}=1$. The mapping $U \rightarrow(a, b)$ is a homeomorphism of $S U(2)$ with the unit sphere in complex 2 -space, i.e. with the 3 -sphere $S^{3}$ in real 4 -space. The natural mapping of $S U(2)$ onto $S U(2) / \mathbb{Z}_{2}$ corresponds to the covering mapping of $S^{3}$ onto $P^{3}$, projective 3 -space, which identifies antipodal points of $S^{3}$. Thus $U(2) / T_{1}$ is (homeomorphic to) $P^{3}$. Choose a triangulation of $P^{3}$ 
and let $X$ be its 2 -skeleton. Then, by definition, $H^{1}(X, \mathbb{Z})$, the first cohomology group of $X$ with integral coefficients, is $H^{1}\left(P^{3}, \mathbb{Z}\right)$. Since $H^{1}\left(P^{3}, \mathbb{Z}\right)=0$ (cf. [13; Theorem 3.9.5, p. 135]), $H^{1}(X, \mathbb{Z})=0$, and each mapping of $X$ into $T_{1}$ is inessential (cf. [13; pp. 72-73, Corollary 7.4.4, p. 302 and remarks following] - we are indebted to LeIF KRISTENSEN for drawing our attention to the identification of $H^{m}(K, G)$ with the homotopy classes of mappings of $K$ into the Eilenberg-MacLane space $K(G, m)$ which allowed us to complete the argument that mappings of $X$ into $U(2)$ are inessential). Now $U(2)$ is homeomorphic to $T_{1} \times S U(2)$ hence to $T_{1} \times S^{3}$. Each mapping of $X$ into $U(2)$ yields, by projection, a mapping into $S^{3}$ which is inessential (since $X$ is a 2-complex) [14; Theorem VI 6, p. 88]. Covering the homotopy of this mapping of $X$ into $S^{3}$ establishes that the mapping of $X$ into $U(2)$ (i.e. $T_{1} \times S^{3}$ ) is homotopic to a mapping into $T_{1}$. Having just noted that mappings of $X$ into $T_{1}$ are inessential, we conclude that mappings of $X$ into $U(2)$ are inessential; so that $\gamma(\mathfrak{Q})=\iota_{0}(\mathfrak{A})$.

We have noted that $p: U(2) \rightarrow U(2) / T_{1}$ has no cross section. If the identity mapping of $X$ onto $X$ could be lifted, this cross section over the 2 -skeleton $X$ of $U(2) / T_{1}$ could be extended to a cross section for the total bundle, since the fibre $T_{1}$ has $\pi_{2}\left(T_{1}\right)=0$ (cf. [29; pp. 148-149]). Thus the identity mapping of $X$ onto $X$ cannot be lifted to $U(2)$, and is an element of $\pi(\mathfrak{A})$ not in $\iota_{0}(\mathfrak{A})$.

Let us denote, now, by $\mathfrak{A}_{m n}$ the $C^{*}$-algebra $\mathscr{A}_{m} \otimes \mathscr{M}_{n}$ where $\mathscr{A}_{m}$ is the algebra $C\left(S^{m}\right), S^{m}$ the $m$-sphere. Since $U(n)$ is homeomorphic to $T_{1} \times S U(n)$ and the natural mapping of $S U(n)$ onto $S U(n) / \mathbb{Z}_{n}$ $\left(\approx U(n) / T_{1}\right)$ is a covering mapping

$$
\pi_{m}(U(n)) \approx \pi_{m}(S U(n)) \approx \pi_{m}\left(S U(n) / \mathbb{Z}_{n}\right) \approx \pi_{m}\left(U(n) / T_{1}\right)
$$

for $m \geqq 2$ from [29; 17.8 , p. $93,21.2(2)$, p. 111, 17.6, p. 92]. Using Bott's Periodicity Theorem [2; Theorem 5, p. 51], we have $\pi_{m}\left(U(n) / T_{1}\right)$ $\left(\approx \pi_{m}(U(n))\right)$ is 0 for $m$ even and $\mathbb{Z}$ for $m$ odd $\neq 1$ when $m<2 n$, while $\pi_{2 n}\left(U(n) / T_{1}\right) \approx \mathbb{Z}_{n !}, \pi_{2 n+1}\left(U(n) / T_{1}\right)$ is $\mathbb{Z}_{2}$ for even $n \geqq 2$ and 0 for odd $n ; \pi_{2 n+2}\left(U(n) / T_{1}\right) \approx \mathbb{Z}_{2}+\mathbb{Z}_{(n+1) !}$ for even $n \geqq 4$ and $\approx \mathbb{Z}_{(n+1) ! / 2}$ for odd $n \geqq 3$ (cf. [31; p. 103, p. 117]). We have noted that

$$
\pi\left(\mathfrak{A}_{m n}\right) / \gamma\left(\mathfrak{A}_{m n}\right) \approx \pi_{m}\left(U(n) / T_{1}\right),
$$

so that the list preceding identifies this quotient for the given $m$ and $n$. In particular, for even $m<2 n, \pi\left(\mathfrak{A}_{m n}\right) / \gamma\left(\mathfrak{A}_{m n}\right)$ is 0 , i.e. $\pi\left(\mathfrak{A}_{m n}\right)=\gamma\left(\mathfrak{A}_{m n}\right)$. Hence in this case, each mapping of $S_{m}$ into $U(n) / T_{1}$ lifts to $U(n)$ and $\pi\left(\mathfrak{A}_{m n}\right)=\iota_{0}\left(\mathfrak{A}_{m n}\right)=\gamma\left(\mathfrak{A}_{m n}\right)$. For odd $m \neq 1$ and $m<2 n$,

$$
\pi\left(\mathfrak{A}_{m n}\right) / \gamma\left(\mathfrak{A}_{m n}\right) \approx \mathbb{Z}
$$

so that $\gamma\left(\mathfrak{A}_{m n}\right) \Phi \pi\left(\mathfrak{A}_{m n}\right)$. We shall note that $\iota_{0}\left(\mathfrak{A}_{m n}\right)=\pi\left(\mathfrak{A}_{m n}\right)$ for $m, n=1,2, \ldots$, by universal bundle techniques. (We are indebted, 
once again, to L. KRISTENSEN for pointing out the use of universal bundle methods in providing a detailed description of mappings which lift.)

We begin by identifying $\iota_{0}(\mathfrak{U})$ more carefully, with $\mathfrak{A}=\mathscr{A} \otimes \mathscr{M}_{n}$ and $\mathscr{A}=C(X)$. Suppose that $X$ is a (compact) $k$-dimensional complex. Let $B$ be an $m$-universal bundle with base $B_{1}$ fibre and group $T_{1}$ and projection $q$ (cf. $[29 ; 19.2$, p. 101, 19.6, p. 103]), where $m$ is taken very large relative to $k$ and $n$. Note that each simplicial mapping $g$ of $X$ into $B$ is inessential, for $G_{0}$ defined on the subcomplex $X \times\{0\} \cup X \times\{1\}$ of $X \times[0,1]$ by $G_{0}(x, 1)=b_{0}$ (a fixed point of $B$ ) and $G_{0}(x, 0)=g(x)$ can be extended to a homotopy $G$, mapping $X \times[0,1]$ into $B$, of $g$ with the constant mapping of $X$ into $b_{0}$, since the high connectivity of $B$ (cf. [29; 19.4 , p. 102]) guarantees that there is no obstruction to the stepwise extension of $G_{0}$ over a simplex of $X \times[0,1]$ of a certain dimension from its value on the boundary of that simplex in the skeleton of $X \times[0,1]$ of one lower dimension.

Since each mapping $g$ of $X$ into $B$ is homotopic to a simplicial mapping (Simplicial Approximation Theorem $[13 ; 1.7 .10$ to 1.8.1, p . 37]), $g$ is inessential. Thus a mapping of $X$ into $B_{1}$ which lifts to $B$ is seen to be inessential by projecting the homotopy of the lifted mapping to a constant mapping into $B$. Conversely if a mapping of $X$ into $B_{1}$ is inessential the Covering Homotopy Theorem $[29 ; 11.7$, p. 54] provides a lifting of it to $B$; so that the mappings of $X$ into $B_{1}$ which can be lifted are precisely the inessential ones.

From the universal property of $B$, there is a bundle mapping $h$ of $U(n)$ into $B$ inducing a mapping $\bar{h}$ of $U(n) / T_{1}$ into $B_{1}$ (cf. $[29 ; 2.5$, p. 9]). Moreover $\left[29 ; \S 10\right.$, pp. 47-49] the bundle $B^{\prime}$ induced by $\bar{h}$ over $U(n) / T_{1}$ is equivalent to $U(n)$ over $U(n) / T_{1}$. Thus the possibility of lifting a mapping $f$ from $X$ into $U(n) / T_{1}$ to $U(n)$ is equivalent to that of lifting $f$ from $U(n) / T_{1}$ to $B^{\prime}$. Now $B^{\prime}$ is the set of points $(u, b)$ in $\left(U(n) / T_{1}\right) \times B$ such that $\bar{h}(u)=q(b)$ (cf. [29; 10.2, p. 47]); so that if $g$ is a lifting of $\bar{h} f$ from $B_{1}$ to $B$, then $f$ defined by $f(x)=(f(x), g(x))$ is a lifting of $f$ from $U(n) / T_{1}$ to $B^{\prime}$ since $\bar{h} f(x)=q g(x)$. Conversely, if $\tilde{f}$ lifts $f$ from $U(n) / T_{1}$ to $B^{\prime}$, then $f(x)=(f(x), g(x))$ for each $x$ in $X$ and some mapping $g$ of $X$ into $B$, since the projection of $B^{\prime}$ onto $U(n) / T_{1}$ is, by construction, projection onto the first coordinate; and $g$ lifts $\bar{h} f$ from $B_{1}$ to $B$ since $\bar{h} f(x)=q g(x)$. Thus $f$ lifts to $U(n)$ if and only if $\bar{h} f$ lifts to $B$, that is, if and only if $\bar{h} f$ is homotopic to a constant mapping into $B_{1}$. With $\mathfrak{A}=\mathscr{A} \otimes \mathscr{M}_{n}$ and $\mathscr{A}=C(X), \iota_{0}(\mathfrak{Q})$ is the group (under pointwise multiplication in $\left.U(n) / T_{1}\right)$ of mappings $f$ of $X$ into $U(n) / T_{1}$ such that $\bar{h} f$ is inessential. In particular, taking $S^{k}$ for $X$ with $k \geqq 3$, we see that all mappings $f$ lie in $\iota_{0}(\mathfrak{A})\left(=\iota_{0}\left(\mathfrak{A}_{k n}\right)\right)$ for $\pi_{k}\left(B_{1}\right) \approx \pi_{k}(B)=0$ (recall that $B$ is $m$-universal, so, $m-1$ connected, with $k<m)$, from the exactness 
of the bundle homotopy sequence $[29 ; 17.4$, p. 91$], \cdots \rightarrow \pi_{k}\left(T_{1}\right)$ $\rightarrow \pi_{k}(B) \rightarrow \pi_{k}\left(B_{1}\right) \rightarrow \pi_{k-1}\left(T_{1}\right)$ and the fact that $\pi_{k-1}\left(T_{1}\right)=\pi_{k}\left(T_{1}\right)$ $=\pi_{k}(B)=0$ with $k \geqq 3$. Since $\pi_{2}\left(U(n) / T_{1}\right)=0$ and the arcwise connectedness of $T_{1}$ allows us to lift mappings of a 1-complex into $U(n) / T_{1}$ to $U(n)$ (as noted earlier when we discussed the case $X=T_{1}$ ), we see that $\iota_{0}\left(\mathfrak{U}_{m n}\right)=\pi\left(\mathfrak{A}_{m n}\right)$ for all $m, n=1,2, \ldots$.

We can show that $\iota_{0}\left(\mathfrak{A}_{m n}\right)=\pi\left(\mathfrak{A}_{m n}\right)$ without universal bundle techniques by a more special analysis. From the homotopy sequence of the bundle,

$$
\cdots \rightarrow \pi_{m}\left(T_{1}\right) \rightarrow \pi_{m}(U(n)) \stackrel{p_{*}}{\longrightarrow} \pi_{m}\left(U(n) / T_{1}\right) \rightarrow \pi_{m-1}\left(T_{1}\right) \rightarrow \cdots ;
$$

so that $p_{*}$ is an isomorphism of $\pi_{m}(U(n))$ onto $\pi_{m}\left(U(n) / T_{1}\right)$ for $m \geqq 3$. For $m=2, \pi_{2}\left(U(n) / T_{1}\right)=0$ as noted earlier. For $m=1, \pi_{0}\left(T_{1}\right)=0$, so that $p_{*}$ is surjective for all $m$. Thus each mapping of $S^{m}$ into $U(n) / T_{1}$ is homotopic to the projection of some mapping of $S^{m}$ into $U(n)$, i.e. homotopic to a mapping which lifts, and hence lifts itself. It follows that $\iota_{0}\left(\mathfrak{U}_{m n}\right)=\pi\left(\mathfrak{A}_{m n}\right)$ for all $m$ and $n$.

\section{References}

[1] Blattiner, R.: Automorphic group representations. Pacific J. Math. 8, $665-677$ (1958).

[2] Вотт, R.: The space of loops on a Lie group. Mich. Math. J. 5, 35-61 (1958).

[3] Borchers, H.: Energy and momentum as observables in quantum field theory. Commun. Math. Phys. 2, 49-54 (1966).

[4] Chevalley, C.: Lie Groups. Princeton: University Press 1946.

[5] Dell'Antonio, G.: On some groups of automorphisms of physical observables. Commun. Math. Phys. 2, 384-397 (1966).

[6] Dixmrer, J.: Les algèbres d'opérateurs dans l'espace hilbertien. Paris: Gauthier-Villars 1957.

[7] - Les $C^{*}$-algèbres et leurs représentations. Paris: Gauthier-Villars 1964.

[8] Dunford, N., and J. Schwartz: Linear operators, Part I. New York: 1958.

[9] GARDNer, L.: An invariance theorem for representations of Banach algebras. Proc. Am. Math. Soc. 16, 983-986 (1965).

[10] Gelfand, I., and M. Neumark: On the imbedding of normed rings into the ring of operators in Hilbert space. Rec. Math. (mat. Sbornik) N.S. 12, 197-213 (1943).

[11] Grtmm, J.: On a certain class of operator algebras. Trans. Am. Math. Soc. 95, 318-340 (1960).

[12] -, and R. Kadison: Unitary operators in $C^{*}$-algebras. Pacific J. Math. 10, $547-556(1960)$.

[13] Hilton, P., and S. Wylie: Homology theory. Cambridge: University Press 1960.

[14] Hurewicz, W., and H. Wallman : Dimension theory. Princeton: University Press 1948.

[15] KaDISON, R.: Unitary invariants for representations of operator algebras. Ann. of Math. 66, 304-379 (1957).

[16] - Derivations of operator algebras. Ann. Math. 83, 280-293 (1966). 
[17] - Transformations of states in operator theory and dynamics. Topology, 3 Suppl. 2, 177-198 (1965).

[18] -, and J. RINGRose: Derivations of operator group algebras. Am. J. Math. 88, 562-576 (1966).

[19] - Automorphisms of operator algebras. Bull. Am. Math. Soc. (to appear).

[20] Kaplansky, I.: Modules over operator algebras. Am. J. Math. 75, 839-859 (1953).

[21] Krein, M., and D. Mrlman: On the extreme points of regular convex sets. Studia Math. 9, 133-137 (1940).

[22] SaKaI, S.: On topological properties of $W^{*}$-algebras. Proc. Japan Acad. 33, $439-444$ (1957).

[23] — On a conjecture of Kaplansky. Tôhoku Math. J. 12, 31-33 (1960).

[24] - Derivations of $W^{*}$-algebras. Ann. Math. 83, 273-279 (1966).

[25] Schwartz, J.: Lectures on $W^{*}$-algebras. NYU notes (mimeographed), 1964.

[26] Segal, I.: Irreducible representations of operator algebras. Bull. Am. Math. Soc. 53, $73-88$ (1947).

[27] Shale, D., and W. Stinespring: States of the Clifford algebra. Ann. Math. 80, 365-381 (1964).

[28] Singer, I.: Automorphisms of finite factors. Am. J. Math. 77, 117-133 (1955).

[29] Steenrod, N.: Fibre bundles. Princeton: University Press 1951.

[30] Suzukr, N.: A linear representation of a countably infinite group. Proc. Japan Acad. 34, 575-579 (1958).

[31] Toda, H.: A topological proof of theorems of Bott and Borel-Hirzebruch for homotopy groups of unitary groups. Mem. Coll. Sci. Univ. Kyoto Ser. A. Math. 32, 103-119 (1959). 